\title{
Redistribution of FLAgellar Member 8 during the trypanosome life cycle: Consequences for cell fate prediction
}

\author{
Estefanía Calvo-Álvarez $^{1,2}$ ( 1 | Serge Bonnefoy ${ }^{1}$ | Audrey Salles ${ }^{3}$ | \\ Fiona E. Benson ${ }^{4}$ | Paul G. McKean ${ }^{4}$ | Philippe Bastin ${ }^{1}$ | Brice Rotureau ${ }^{1,2}$
}

\author{
${ }^{1}$ Trypanosome Cell Biology Unit, Institut \\ Pasteur and INSERM U1201, Paris, France \\ ${ }^{2}$ Trypanosome Transmission Group, Institut \\ Pasteur, Paris, France \\ ${ }^{3}$ Unit of Technology and Service Photonic \\ Biolmaging (UTechS PBI), C2RT, Institut \\ Pasteur, Paris, France \\ ${ }^{4}$ Division of Biomedical and Life Sciences, \\ Lancaster University, Lancaster, UK

\section{Correspondence} \\ Estefanía Calvo Alvarez, Trypanosome Cell \\ Biology Unit, INSERM U1201, Institut Pasteur, \\ Paris, France. \\ Email: estefaniacalvoalvarez@gmail.com \\ Funding information \\ French National Research Agency, Grant/ \\ Award Number: ANR-10-INSB-04; French \\ National Agency for Scientific Research, \\ Grant/Award Numbers: ANR-18-CE15-0012 \\ TrypaDerm, ANR-14-CE14-0019-01 EnTrypa; \\ French Government Investissement d'Avenir \\ Programme, Grant/Award Number: ANR- \\ 10-LABX-62-IBEID; Institut National pour la \\ Santé et le Recherche Médicale (INSERM); \\ Institut Pasteur
}

\begin{abstract}
The single flagellum of African trypanosomes is essential in multiple aspects of the parasites' development. The FLAgellar Member 8 protein (FLAM8), localised to the tip of the flagellum in cultured insect forms of Trypanosoma brucei, was identified as a marker of the locking event that controls flagellum length. Here, we investigated whether FLAM8 could also reflect the flagellum maturation state in other parasite cycle stages. We observed that FLAM8 distribution extended along the entire flagellar cytoskeleton in mammalian-infective forms. Then, a rapid FLAM8 concentration to the distal tip occurs during differentiation into early insect forms, illustrating the remodelling of an existing flagellum. In the tsetse cardia, FLAM8 further localises to the entire length of the new flagellum during an asymmetric division. Strikingly, in parasites dividing in the tsetse midgut and in the salivary glands, the amount and distribution of FLAM8 in the new flagellum were seen to predict the daughter cell fate. We propose and discuss how FLAM8 could be considered a meta-marker of the flagellum stage and maturation state in trypanosomes.
\end{abstract}

\section{KEYWORDS}

differentiation, division, elongation, flagellum, FLAM8, remodelling, Trypanosoma brucei

\section{1 | INTRODUCTION}

Trypanosoma brucei is an extracellular parasite responsible for African trypanosomiases, also known as sleeping sickness in humans and nagana in cattle. African trypanosomes are blood and tissue-dwelling protists transmitted to their mammalian hosts by the bite of the blood-feeding tsetse fly (Glossina genus) in sub-Saharan Africa. Trypanosoma brucei is subjected to a complex developmental cycle highly organised in space and time, and characterised by the existence of multiple stages alternating between the two hosts (Rotureau \& Van Den Abbeele, 2013).

The T. brucei flagellum is an essential organelle anchored along the surface of the cell body and present in all stages of its development (Rotureau, Subota, \& Bastin, 2011). It is essential for parasite viability (Broadhead et al., 2006), cell division and morphogenesis (Kohl, Robinson, \& Bastin, 2003), attachment to the tsetse salivary glands (Tetley \& Vickerman, 1985) and motility (Rotureau, Ooi, Huet, Perrot, \& Bastin, 2014; Shimogawa et al., 2018), but it also possibly contributes to sensory functions and interactions with the microenvironment (Roditi, Schumann, \& Naguleswaran, 2016; Rotureau, Morales, Bastin, \& Spath, 2009). The trypanosome flagellum is composed of a canonical axoneme containing nine doublet microtubules and a central pair of singlet microtubules, associated with a paraflagellar rod (PFR) and surrounded by a specialised membrane (Langousis \& Hill, 2014). This single flagellum exits the cytoplasm from the flagellar pocket, a specialised membrane invagination (Field \& 
Carrington, 2009). In T. brucei, the flagellum remains attached along most of its length to the cell body by the flagellum attachment zone with the exception of its distal end that extends to a short distance beyond the cell body (Taylor \& Godfrey, 1969).

Unlike the microtubule-based core and the proximal basal body, our understanding of the molecular composition, specific structures and functions of the distal tip region is still limited, due mainly to its small dimensions and its continuity with other flagellar structural components. During flagellum construction, incorporation of axonemal precursors and PFR subunits only takes place at the distal tip of the growing flagellum (Bastin, MacRae, Francis, Matthews, \& Gull, 1999; Sherwin, Schneider, Sasse, Seebeck, \& Gull, 1987), which exhibits a complex ultrastructure that differs between insect and mammalian forms (Hoog et al., 2014; Hughes, Towers, Starborg, Gull, \& Vaughan, 2013). In addition, the IntraFlagellar Transport (IFT) machinery mediates delivery of nascent flagellum subunits towards the ciliary tip for assembly into the growing flagellum and back to its base (Buisson et al., 2013). At the tip, the canonical axoneme organisation collapses as microtubules are attached together in a resistive structure that prevents microtubule sliding but permits bend formation, which results in tip-to-base flagellar beating (Heddergott et al., 2012; Woolley, Gadelha, \& Gull, 2006). Several studies have investigated the presence of discrete proteins that localise to the distal tip in trypanosomes, suggesting the existence of a specific subdomain (Chan \& Ersfeld, 2010; Liu, Apagyi, McLeavy, \& Ersfeld, 2010; Saada et al., 2014; Varga, Moreira-Leite, Portman, \& Gull, 2017; Woodward, Carden, \& Gull, 1995).

Our proteomic analysis of preserved flagella purified from the insect midgut procyclic forms of $T$. brucei parasite (PCF) identified a group of flagellar membrane and matrix proteins with unique patterns and dynamics (Subota et al., 2014). Among them, one protein termed FLAgellar Member 8 (FLAM8) is present only at the distal tip of the flagellum of PCF grown in culture. This large protein (3,075 amino acids) is progressively added to the new flagellum during its assembly (Subota et al., 2014) and requires IFT to be maintained at the distal tip (Fort, Bonnefoy, Kohl, \& Bastin, 2016). In PCF trypanosomes, FLAM8 concentrates at the tip of axonemal microtubules after detergent extraction, (Subota et al., 2014) demonstrating its strong association to the flagellum cytoskeleton and/or an association to other specific structural complexes linked to the flagellum tip. Prior to PCF cell division, FLAM8 distribution in the distal part of the new organelle reaches about one-third of that in the old flagellum (Subota et al., 2014). After cytokinesis, the amount of FLAM8 further increases until the flagellum reaches its final length. Recently, a new model termed 'grow and lock' described how the new flagellum elongates until a locking event fixes the final length in a timely defined manner (Bertiaux, Morga, Blisnick, Rotureau, \& Bastin, 2018). This study identified FLAM8 as a marker of the locking event that controls flagellum length and defines a flagellum that has reached its maturity status.

The grow-and-lock model results from observations in PCF cells cycling in stable culture conditions and that produce the same type of progeny. However, within each host, trypanosomes have to face different micro-environments, which requires major morphological and

\section{Take Away}

- FLAM8 extends along the entire flagellar cytoskeleton in mammalian infective forms and concentrates to the distal end during differentiation into the insect procyclic stage.

- In parasites dividing in the tsetse midgut, the amount and distribution of FLAM8 in the new flagellum predicts the daughter cell fate, either towards another round of proliferation or towards flagellum elongation and next cell differentiation.

- The amount of FLAM8 in the new flagellum of 'EpiTrypo' dividing cells in the salivary glands predicts the pre-metacyclic daughter cell fate.

- In total, FLAM8 could be considered a meta-marker of the flagellum stage and maturation state in trypanosomes.

metabolic adaptations, driven by the activation of specific gene expression programmes that are critical for life-cycle progression (MacGregor, Szoor, Savill, \& Matthews, 2012; Ooi \& Bastin, 2013; Smith, Bringaud, Nolan, \& Figueiredo, 2017). These drastic changes are also true for flagella that evolve in length, position and shape (Ooi \& Bastin, 2013; Rotureau et al., 2011) as well as in molecular composition (Oberholzer et al., 2011; Rotureau, Subota, Buisson, \& Bastin, 2012; Subota et al., 2014). We reasoned that FLAM8 location and functions could also evolve during the parasite cycle. Especially, the role of FLAM8 as a marker of maturity at the tip of the flagellum could differ among specific stages, and FLAM8 could participate in flagellum length control in different ways through the trypanosome development.

Here, we detail the differential FLAM8 localisation profiles over both the cell and parasite cycles in vivo in mammalian and insect hosts. FLAM8 amount and distribution along the T. brucei flagellum are stage-specific. The first FLAM8 redistribution during the mammalian to insect form differentiation in the tsetse midgut illustrates the remodelling of an existing flagellum, while the second redistribution takes place during the construction of the new short flagellum in long asymmetrically dividing epimastigotes of the cardia. FLAM8 appears as a marker of the flagellum maturation state that could predict the fate of procyclic trypomastigotes dividing in the midgut and attached epimastigotes dividing in the salivary glands.

\section{2 | RESULTS}

\subsection{FLAM8 is localised along the entire flagellum in BSF}

Two trypanosome stages can be found in the bloodstream of a mammalian host: the dividing slender (SL) bloodstream form (BSF) and the non-proliferative stumpy (ST) form pre-adapted for transmission to 
the insect vector. To study the subcellular localisation of FLAM8 in BSF trypanosomes, an anti-GFP antibody was used for immunostaining in combination with the mAb25 axonemal marker in parasites expressing FLAM8 coupled to EYFP at the $\mathrm{N}$-terminal end. In contrast to PCF cells, FLAM8 was not only present at the distal tip of the flagellum, but it was also distributed along the entire flagellum length, with an enrichment in the proximal half of the flagellum in BSF in culture (Figure 1a). At the BSF flagellum base, FLAM8 was detected after the emergence of the axoneme from the transition zone but before the beginning of the PFR, possibly at the level of the flagellar pocket collar, evidenced by the use of the flagellar transition zone component (FTZC; Bringaud et al., 2000) and PFR markers in BSF parasites expressing EYFP::FLAM8 (Figure 1b).

Trypanosoma brucei arginine kinase 3 (AK3) is localised to the entire flagellum membrane in all stages of parasite development in the tsetse fly as well as in BSF parasites (Ooi et al., 2015), representing a robust flagellum membrane marker. To assess whether FLAM8 could be associated with the BSF flagellum membrane, an antiserum raised against the arginine kinase of Trypanosoma cruzi (anti-TcAK; Pereira, Alonso, Torres, \& Flawia, 2002; Subota et al., 2014), was used for immunofluorescence assay in combination with the anti-GFP antibody in parasites expressing EYFP::FLAM8 either on methanol-fixed or paraformaldehyde-fixed whole cells, or detergent-extracted cytoskeletons. Distinct fluorescent signals were observed for FLAM8 and AK3 in the flagellum of parasites fixed in methanol and PFA-fixed (Figure 1c, left and middle panels). This absence of overlap suggests that FLAM8 is not associated with the flagellum membrane. This was confirmed by the presence of FLAM 8 at the proximal part of the flagellum and the distal tip after detergent extraction, whereas membrane removal led to a complete loss of the anti-AK3 signal (Figure 1c, right panels). In order to define more closely the localisation of FLAM8 to the flagellum cytoskeleton, structured illumination microscopy (SIM) was employed with anti-GFP for FLAM8 staining, and markers for the axoneme and the PFR (Kohl, Sherwin, \& Gull, 1999) in both EYFP::FLAM8 PCF and SL BSF (Figure 1d). In PCF, the triple staining revealed that FLAM8 is exclusively located at the distal end of the flagellum in growing and mature flagella (Figure 1d, upper panels). In contrast, bloodstream parasites exhibit a specific FLAM8 proximal localisation that starts at the base of the flagellum and extends towards the half of the flagellum length in both old and new flagella (Figure 1d, lower panels). At this point, the signal diminishes until reaching the distal tip of the cilium, where a bright FLAM8 (a)
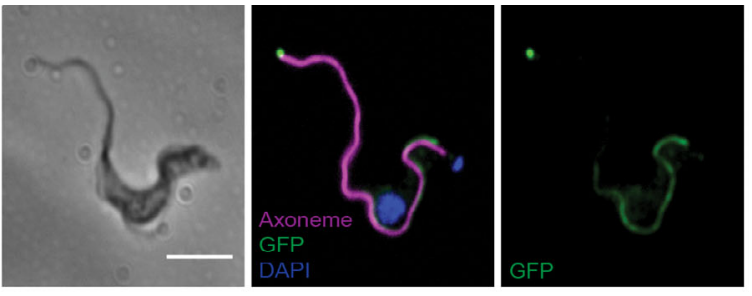

(b)
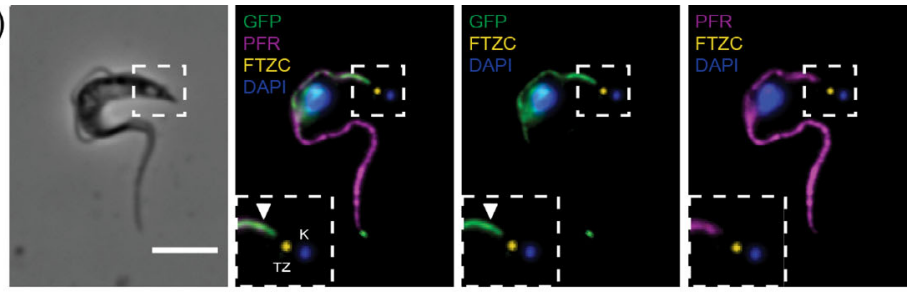

(c)
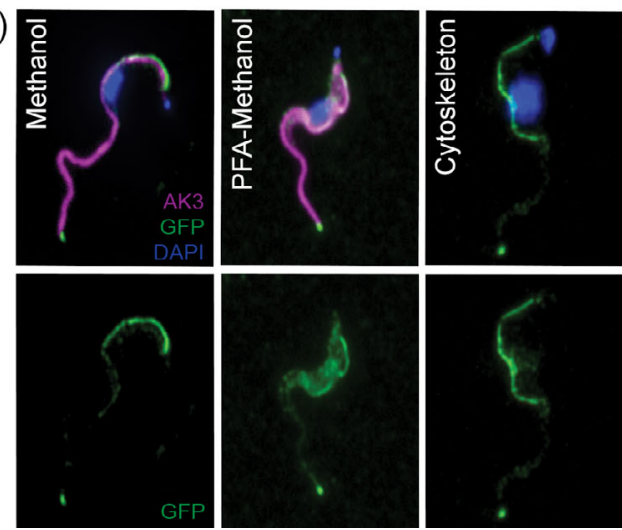
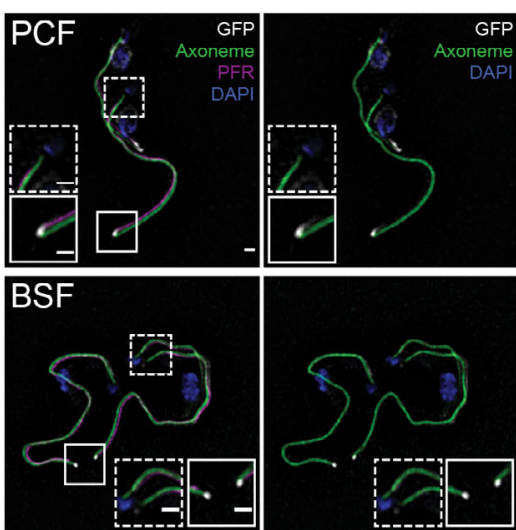
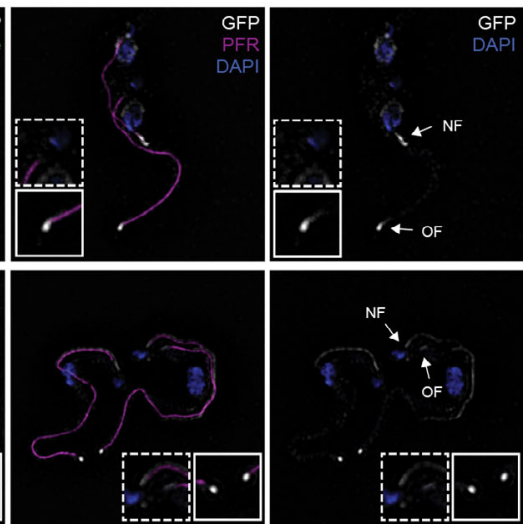

FIGURE 1 FLAM8 is differentially distributed along the entire flagellum in mammalian forms. (a) Immunofluorescence on methanol-fixed AnTat EYFP::FLAM8 BSF in culture, using the anti-GFP (green), the anti-axoneme marker mAb25 (magenta), DAPI staining of DNA content (blue). Scale bar is $5 \mu \mathrm{m}$. (b) IFA on methanol-fixed AnTat EYFP::FLAM8 BSF showing that the base of the flagellum FLAM8 (anti-GFP signal in green) do not co-localise with the transition zone component FTZC (in yellow) and initiates before the starting of the PFR (in magenta). White arrowheads indicate the beginning of the PFR signal. Scale bar is $5 \mu \mathrm{m}$. (c) FLAM8 is not localised to the flagellum membrane. IFA on methanolfixed AnTat EYFP::FLAM8 BSF (left panels), paraformaldehyde-fixed (middle panels) and cytoskeletons (right panels) using the anti-GFP antibody (green) and an antiserum against the arginine kinase of T. cruzi (magenta), DAPI staining of DNA content (blue). Arginine kinase (AK) signal, which is localised to the flagellum membrane, was almost completely lost after detergent extraction (right panels). Scale bar is $5 \mu \mathrm{m}$. (d) Structured illumination microscopy images (SIM) of FLAM8 (anti-GFP antibody in white) with flagellar components, paraflagellar rod (PFR, magenta) and axoneme (green) showed in pleomorphic EYFP::FLAM8 PCF and BSF parasites (upper and lower panels, respectively). DAPI staining of DNA content appears in blue. Scale bars represent $1 \mu \mathrm{m}$. Images: white arrows show the old (OF) and the new flagellum (NF). Images are representative of three independent experiments for panels (a), (b) and (c), and two independent experiments for panel (d) 
accumulation is evidenced (Figure 1d, lower panels). In both PCF and BSF, and in both mature and growing flagella, FLAM8 is distinct from, but resides in the vicinity of the axoneme and the PFR (Figure 1d, upper and lower panels). This was observed in multiple cells and after calibration of the SIM settings with fluorescent microspheres to prevent any pixel shift. At the distal end, FLAM8 signal appears more extended and diffuse in PCF compared to the rounded and more defined shape observed in BSF. In addition, in the proximal region of the BSF flagellum only, SIM images confirm that FLAM8 signal initiates after the axoneme but before the PFR.

\subsection{FLAM8 is a marker of flagellum maturation in BSF}

African trypanosomes possess a nucleus $(\mathrm{N})$ and a single mitochondrion whose genetic material is condensed in a structure named the kinetoplast $(K)$ and that is physically linked to the basal body of the flagellum (Robinson \& Gull, 1991). The progression through the cell cycle can be monitored by staining DNA content as parasites with one kinetoplast and one nucleus $(1 \mathrm{~K} 1 \mathrm{~N})$ are in the $\mathrm{G} 1 / \mathrm{S}$ phase, those with two kinetoplasts and one nucleus (2K1N) are in G2/M and cells with two kinetoplasts and two nuclei $(2 \mathrm{~K} 2 \mathrm{~N})$ are about to undergo cytokinesis (Sherwin \& Gull, 1989; Woodward \& Gull, 1990). FLAM8 was described as a molecular marker for flagellum maturation in cultured PCF as a maximum amount of FLAM8 is found in flagella that do not elongate anymore after having reached their mature state (Bertiaux et al., 2018). This led us to explore whether FLAM8 would have a similar behaviour in BSF trypanosomes in vitro and in vivo (Figure 2 and Figure S2). In order to perform quantitative analyses in flagella, both at the distal tip and at the proximal region, we used BSF trypanosomes expressing EYFP:: FLAM8. The portion of the proximal region containing FLAM8 was measured in mature and growing flagella following a double immunofluorescence assay with an anti-GFP and the mAb25 axonemal marker for measurements. In $1 \mathrm{~K} 1 \mathrm{~N}$ parasites, FLAM8 was observed in the first $13.5 \mu \mathrm{m} \pm 2.0 \mu \mathrm{m}$ of the proximal region, representing $52 \%$ of the flagellum length, as well as at the distal tip of the mature flagellum (Figure 2a, left panels and $2 \mathrm{~B}$ ). In $1 \mathrm{~K} 1 \mathrm{~N}$ parasites containing a short new flagellum and in $2 \mathrm{~K} 1 \mathrm{~N}$ trypanosomes undergoing mitosis, the presence of FLAM8 was restricted to the first $5.9 \mu \mathrm{m}$ $\pm 3.2 \mu \mathrm{m}$ and $11.6 \mu \mathrm{m} \pm 3.1 \mu \mathrm{m}$ of the proximal region of the new flagellum, respectively (Figure $2 \mathrm{a}$, middle panels), which corresponded to 71 and $75 \%$ of the length of the growing flagellum, respectively (Figure $2 \mathrm{~b}$ ). The FLAM8 distribution further extended up to $15.1 \mu \mathrm{m} \pm 3.8 \mu \mathrm{m}$ in length in the new flagellum of $2 \mathrm{~K} 2 \mathrm{~N}$ cells (Figure 2a, right panels and $2 \mathrm{~B}$ ). At this point, the new flagellum has reached about $80 \%$ of its final mature length (not shown), implying that FLAM8 should still accumulate and/or re-locate along the full flagellum length after cell division until the growing flagellum has reached a definitive length and before the cell re-enters a new cell cycle. There was a linear correlation between the length of FLAM8 distribution in the proximal region and the total length of the axoneme at all steps of the cell cycle, except in the new flagella of $2 \mathrm{~K} 2 \mathrm{~N}$ cells, where a less defined pattern was observed (Figure $2 \mathrm{c}$ ).

To investigate whether FLAM8 distribution is dynamic and cellcycle dependent in BSF as it is the case in PCF, cell division was inhibited by treating pleomorphic EYFP::FLAM8 BSF cells with $10 \mu \mathrm{M}$ teniposide, a topoisomerase II inhibitor that interferes with mitochondrial DNA segregation but neither with basal body duplication nor with flagellum elongation (Robinson \& Gull, 1991). In untreated cells, FLAM 8 was abundant at the tip and in the proximal region of the old flagellum and present in lower amounts in the new flagellum (Figure 2d, upper panels). By contrast, in cells treated with teniposide for $6 \mathrm{hr}$ (just under the cell doubling time), the new flagellum had elongated further and the FLAM8 signal at the distal region was brighter and looked similar to that at the tip of the mature flagellum (Figure 2d, bottom panels). Unfortunately, the length of both old and new flagella could not be quantified due to a frequent overlapping between them in most cells after teniposide treatment. Next, regions of interest were defined around the tip of the new and old flagella, and the total amount of fluorescence was quantified. In untreated cells at an advanced stage of the cell cycle $(2 \mathrm{~K} 2 \mathrm{~N})$, the ratio of the total FLAM8 fluorescence intensity between the new and the old flagella was $80 \%$ (Figure 2e). However, in cells treated with teniposide, this ratio significantly increased up to $100 \%$ (Figure $2 \mathrm{e}$ ), demonstrating that a maximum amount of FLAM8 marker is found in flagella that have reached their final length in BSF trypanosomes as well. This confirms that FLAM8 can be considered a flagellum maturation marker both in PCF and BSF trypanosomes.

\subsection{FLAM8 redistribution occurs in the existing flagellum during BSF to PCF differentiation}

Several hours after the ingestion of a bloodmeal, ST differentiate into PCF parasites in the posterior midgut of the tsetse fly where they start to proliferate again while the digestion occurs. Since FLAM8 presents distinct distributions in BSF and PCF, we investigated how this transition could occur (Figure 3). FLAM8 could be redistributed in non-dividing ST, or alternatively during either the SL to ST or the ST to PCF differentiation. In order to get insights into this remodelling, blood was sampled from the first peak of parasitemia of mice infected with trypanosomes and the redistribution of FLAM8 was investigated. The presence of stumpy cells was assessed by using the anti-PAD1 antibody marker that targets a unique surface transporter (Dean, Marchetti, Kirk, \& Matthews, 2009). The concomitant use of the antiFLAM8 antibody showed that FLAM8 distributed along the full-length flagellum while exhibiting a bright signal at the distal tip in freshly isolated ST cells, as in SL trypanosomes (Figure 3a, left panels). Then, the differentiation of these ST parasites into PCF was induced in vitro by a temperature shift from 37 to $27^{\circ} \mathrm{C}$ coupled to the addition of cisaconitate to the medium (Engstler \& Boshart, 2004; Szoor, Ruberto, Burchmore, \& Matthews, 2010). The emergence of PCF cells was assessed by using an anti-EP-procyclin marker antibody labelling only the procyclin surface coat (Acosta-Serrano et al., 2001). The 
(a)
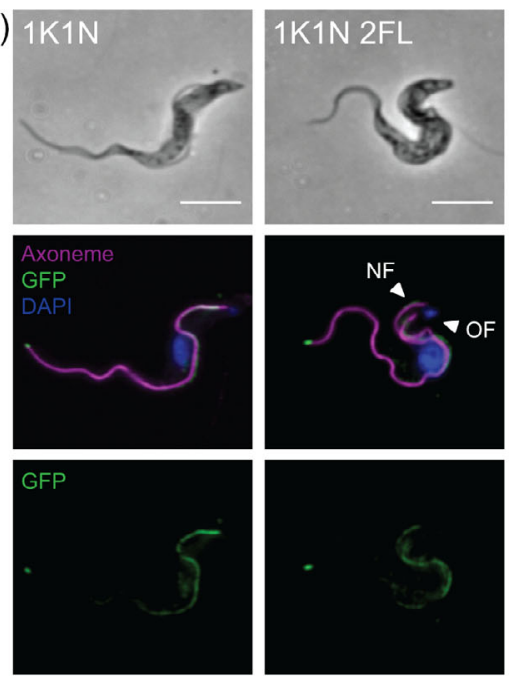
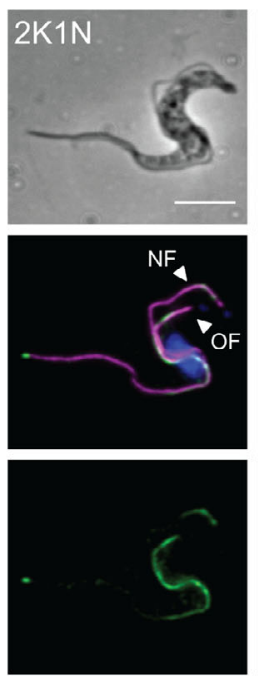
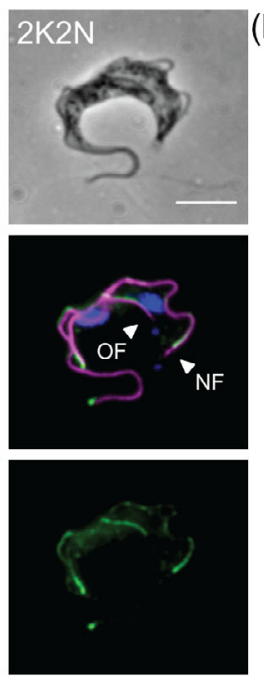

(b)

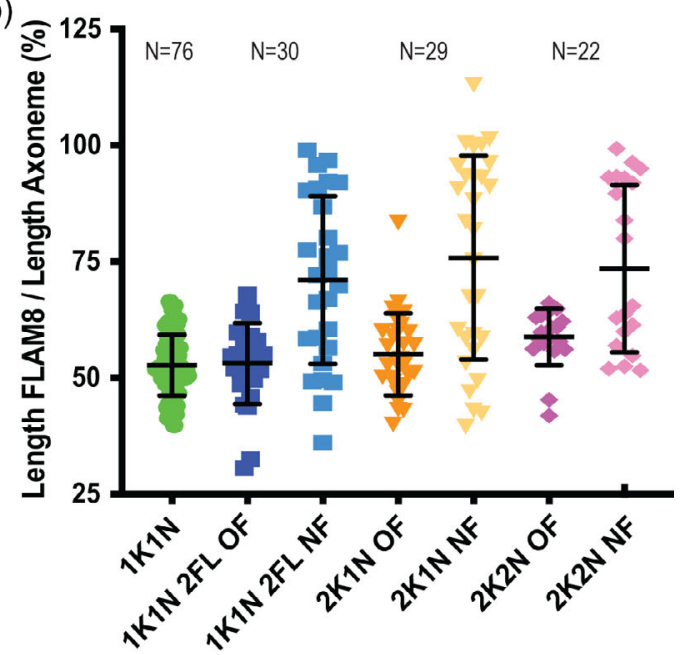

(c)

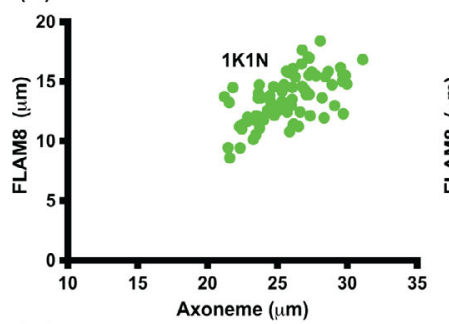

(d)
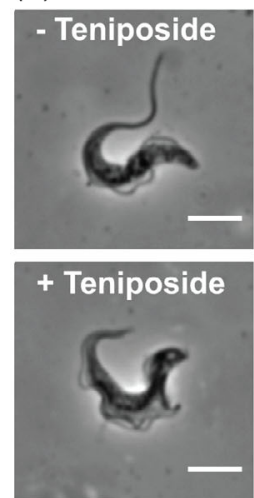

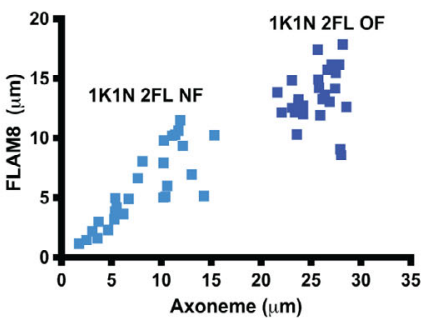

Axoneme $(\mu \mathrm{m})$
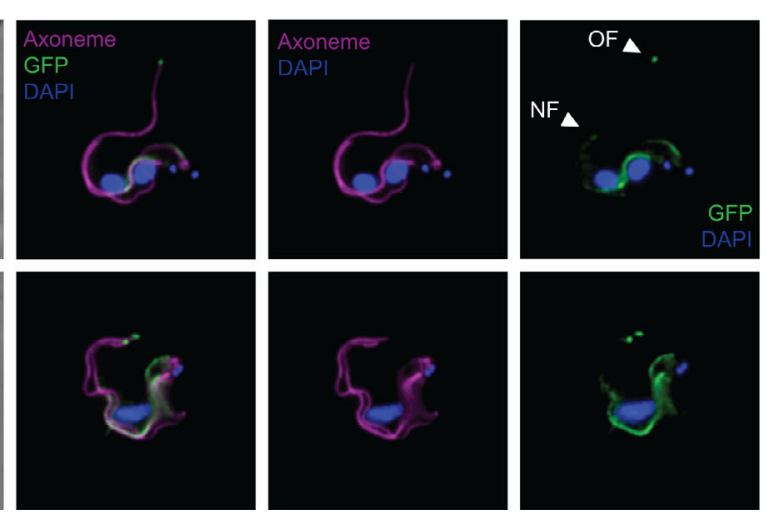

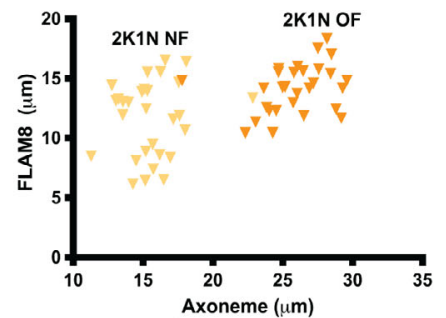

(e)
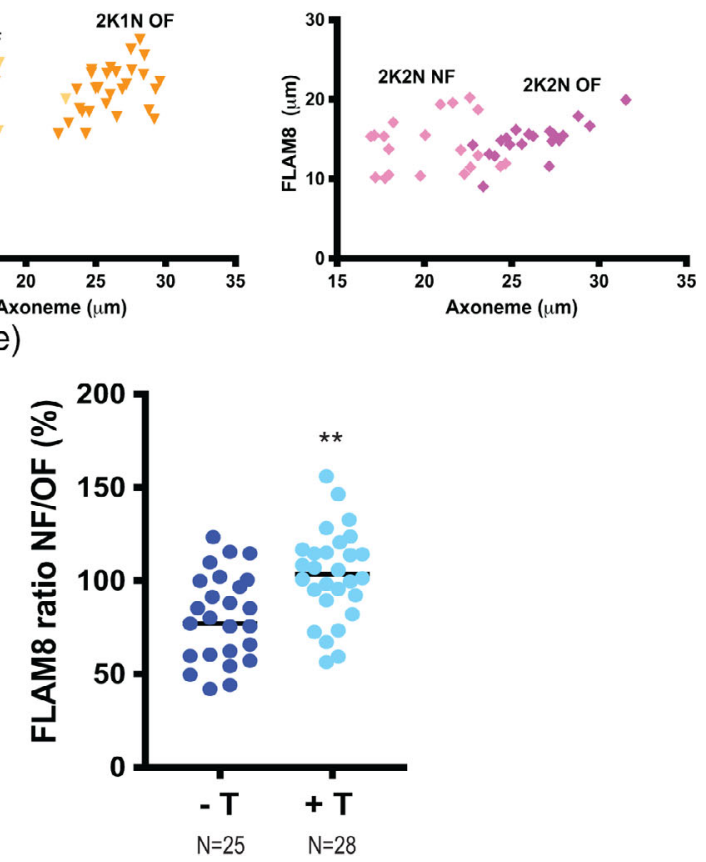

FIGURE 2 FLAM8 is also a marker of the flagellum maturation in mammalian infective forms. (a) Immunofluorescence on AnTat EYFP::

FLAM8 BSF in culture expressing FLAM8 fused to the EYFP protein in the N-terminus. The axoneme (mAb25 antibody in magenta) shows the full length of the flagellum; FLAM8 was labelled using the anti-GFP antibody (green), and DAPI staining of DNA content appears in blue. (b) Dot plot showing the quantification of FLAM8 intensity present at the proximal region in $1 \mathrm{~K} 1 \mathrm{~N}, 1 \mathrm{~K} 1 \mathrm{~N}$ with two flagella (1K1N $2 \mathrm{FL}), 2 \mathrm{~K} 1 \mathrm{~N}$ and $2 \mathrm{~K} 2 \mathrm{~N}$ BSF parasites, compared with the total length of the flagellum measured by the use of the axoneme marker mAb25. The number of cells used for the quantification $(\mathrm{N})$ is indicated above the graph. (c) Linear correlation between the FLAM8 signal and the length of the axoneme in $1 \mathrm{~K} 1 \mathrm{~N}, 1 \mathrm{~K} 1 \mathrm{~N}$ 2FL, 2K1N and 2K2N trypanosomes. (d) IFA on methanol-fixed AnTat EYFP::FLAM8 parasites left untreated (top panels) or treated for $6 \mathrm{hr}$ with teniposide (bottom panels) stained with anti-GFP (green), mAb25 antibody (magenta) and DAPI labelling DNA (blue). (e) Ratio between the FLAM8 fluorescent signal at the tip in the new and old flagellum in cells treated or non treated with teniposide for $6 \mathrm{hr}$. Statistically significant differences upon linear regression analysis followed by unpaired $t$-test are indicated with two stars $(p<.005)$, and the number of cells used for quantification is indicated below the graph. Images: white arrowheads show the old (OF) and the new flagellum (NF). Scale bar is $5 \mu \mathrm{m}$. Results are given as means ( \pm standard deviation, $S D$ ) of biometric measurements of cells from two independent experiments

expression of procyclin at the parasite surface was observed as soon as $2 \mathrm{hr}$ after differentiation (Figures $2 \mathrm{~h}$ and $3 \mathrm{a}$-middle panels). This was concomitant with the FLAM8 enrichment to the distal tip, especially in cells with an intermediate morphology between BSF and PCF (Figure 3a: 2, 4 and $12 \mathrm{hr}$-middle panels). This remodelling culminated with the disappearance of the FLAM 8 proximal signal and its concentration solely at the flagellum tip $24 \mathrm{hr}$ post-differentiation (Figure 3a, right $24 \mathrm{hr}$-panels). The quantification of the FLAM8 signal at the distal tip in SL and ST BSF as compared to early PCF demonstrated the accumulation of the protein at the flagellum tip in PCF parasites (Figure 3b). This accumulation of FLAM8 at the distal tip was also confirmed by repeating the same experiment with in vitro 
(a) SL
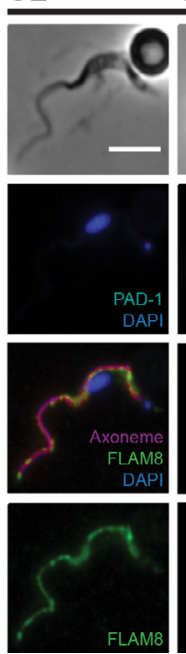

ST
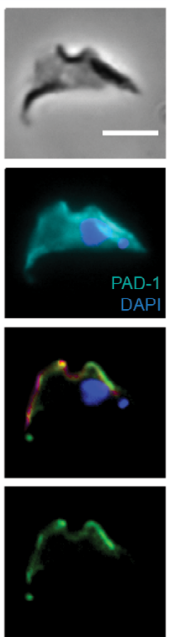

ePCF $2 \mathrm{~h}$

$4 \mathrm{~h}$ $12 \mathrm{~h}$ $24 \mathrm{~h}$

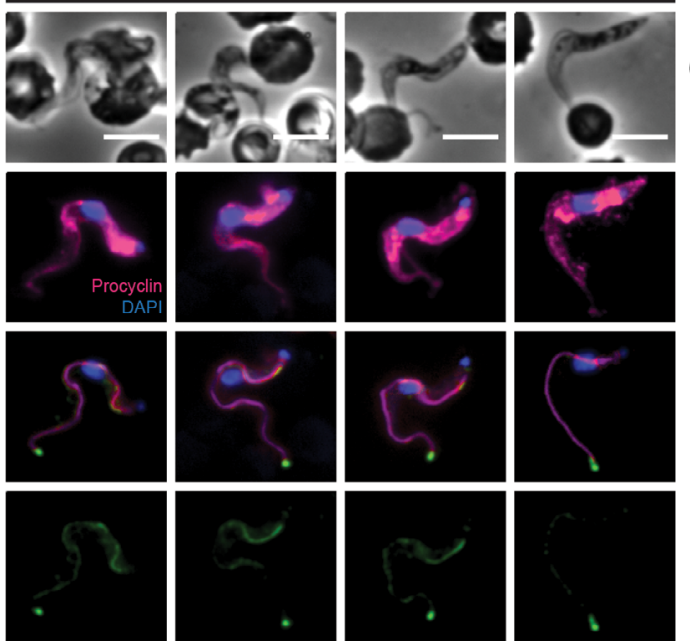

(b)

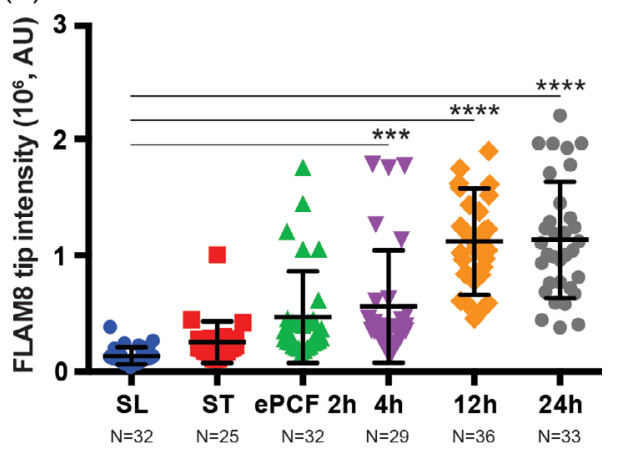

FIGURE 3 FLAM8 concentration to the tip reflects the remodelling of an existing flagellum during BSF to PCF differentiation.

(a) Representative images of AnTat 1.1E SL and ST parasites isolated from infected BALB/c mice and undergoing ex vivo differentiation from non-dividing stumpy forms (left panels) into early procyclic trypanosomes at $27^{\circ} \mathrm{C}$ (panels named 2, 4, 12 and $24 \mathrm{hr}$ ). Immunofluorescence images of methanol-fixed parasites stained with anti-FLAM8 antibody (green), anti-axonemal mAb25 (magenta), and antibodies against specific surface markers: anti-PAD1 to stain ST forms (in cyan), and anti-procyclin labelling early PCF parasites (in pink). DAPI staining appears in blue. Scale bar is $5 \mu \mathrm{m}$. (b) Quantification of the FLAM8 intensity at the tip of the flagellum during ex vivo differentiation into insect procyclic forms. Statistically significant differences after one-way ANOVA followed by Tukey-Kramer post-test are indicated with three $(p<.001)$ and four stars $(p<.0001)$. The number of parasites used for quantification $(\mathrm{N})$ is indicated below the graph. Results are given as means ( \pm standard deviation, $S D$ ) of biometric measurements of cells from one experimental infection and two biological replicates. AU, arbitrary units

induced stumpy form parasites (Figure S3a,b). These modifications of FLAM8 location during ST BSF to PCF differentiation illustrates the remodelling of an existing flagellum in a non-dividing cell.

\subsection{FLAM8 is a marker of cell fate after PCF division}

During the first 3 days after their initial differentiation, PCF proliferate and eventually establish an infection in the posterior midgut (Gibson \& Bailey, 2003). Later, only a subpopulation of these proliferative procyclic parasites progressively elongate and migrate to the anterior part of the midgut as long trypomastigotes, while the others stay in the posterior midgut and maintain the population (Van Den Abbeele, Claes, van Bockstaele, Le Ray, \& Coosemans, 1999). Assuming that PCF division results in the production of two daughter cells that are not equivalent (Abeywickrema et al., 2019), one could propose at least two hypotheses. First, only the daughter cell inheriting the new flagellum would be able to progress in the cyclical development (Ooi \& Bastin, 2013). Second, alternatively or in addition, there could be two distinct types of PCF divisions: one division type leading to two daughter cells intended to further proliferate, and another division type producing one sibling adapted for progressing in the parasite cycle and a second sibling committed for another round of proliferation. In PCF parasites in culture that could virtually proliferate indefinitely, FLAM8 is progressively added to the new flagellum during its construction in $\mathrm{G} 2 / \mathrm{M}$ phase and after cell division in $\mathrm{G} 1$ phase
(Bertiaux et al., 2018; Subota et al., 2014). In order to confirm this in vivo and to explore the two hypotheses, we scrutinised the localisation of FLAM8 in dividing PCF isolated from both the posterior and anterior regions of the tsetse midgut (Figure 4) and treated them for immuno-fluorescence analysis (IFA) with the anti-FLAM8 antibody and the axonemal marker mAb25 (Dacheux et al., 2012). The FLAM8 signal intensity at the distal tip of old flagella (OF) in $1 \mathrm{~K} 1 \mathrm{~N}, 2 \mathrm{~K} 1 \mathrm{~N}$ or $2 \mathrm{~K} 2 \mathrm{~N}$ cells was quantified and compared to that of new flagella (NF) in dividing $2 \mathrm{~K} 1 \mathrm{~N}$ and $2 \mathrm{~K} 2 \mathrm{~N}$ parasites, and the flagellum length was also measured (Figure 4). In recently differentiated PCF isolated from the posterior midgut ( 3 days after the flies received an infective blood meal), a progressive accumulation of FLAM8 was observed in the growing flagellum of $2 \mathrm{~K} 1 \mathrm{~N}$ and $2 \mathrm{~K} 2 \mathrm{~N}$ parasites (Figure $4 \mathrm{a}$ ). The total amount of FLAM8 at the tip of the new flagellum was significantly lower than that in the mature flagellum in $2 \mathrm{~K} 1 \mathrm{~N}$ cells (Figure 4b). A similar yet non-significant trend was also evidenced in $2 \mathrm{~K} 2 \mathrm{~N}$ cells (Figure $4 \mathrm{~b}, \mathrm{c}$ ). This demonstrates that in procyclic trypanosomes from the posterior midgut, FLAM8 addition to the distal tip occurs during the assembly of the new flagellum and that the protein reaches its maximum amount in the new flagellum after cell division, as observed in PCF in culture (Bertiaux et al., 2018; Subota et al., 2014). Nevertheless, a different situation was observed in late PCF extracted from the anterior midgut (Figure 4d,f). Eleven days after ingestion of the blood meal, trypanosomes had migrated and colonised the anterior region, where they were found in high numbers. A region of interest was defined around the tip of all flagella in $1 \mathrm{~K} 1 \mathrm{~N}, 2 \mathrm{~K} 1 \mathrm{~N}$ and $2 \mathrm{~K} 2 \mathrm{~N}$ cells from the anterior midgut, and the total 
(a)
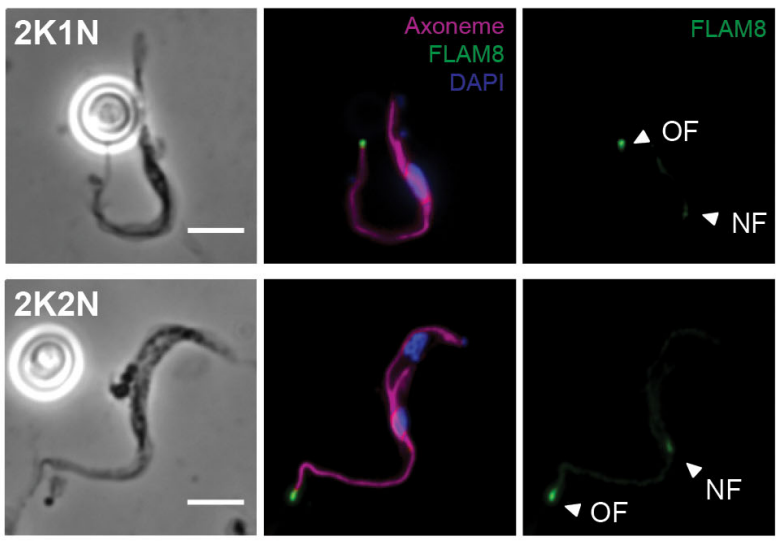

(b)

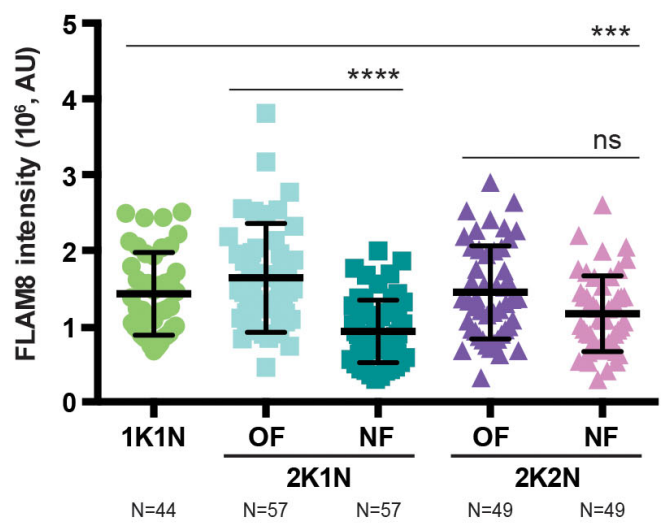

(c)

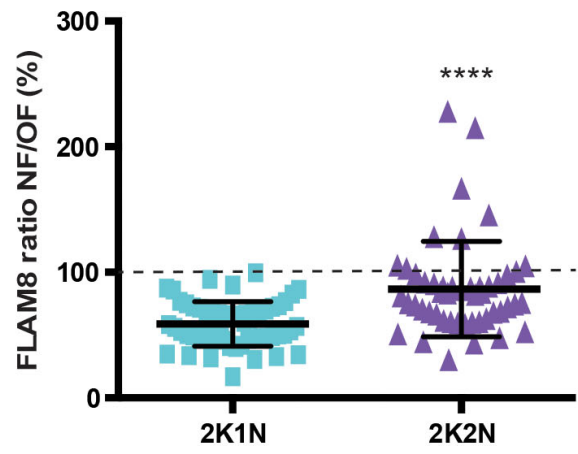

(d)
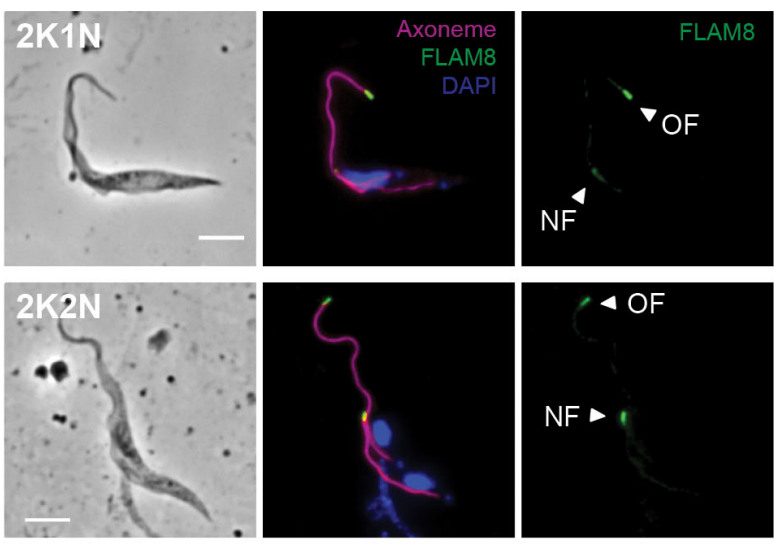

(e)

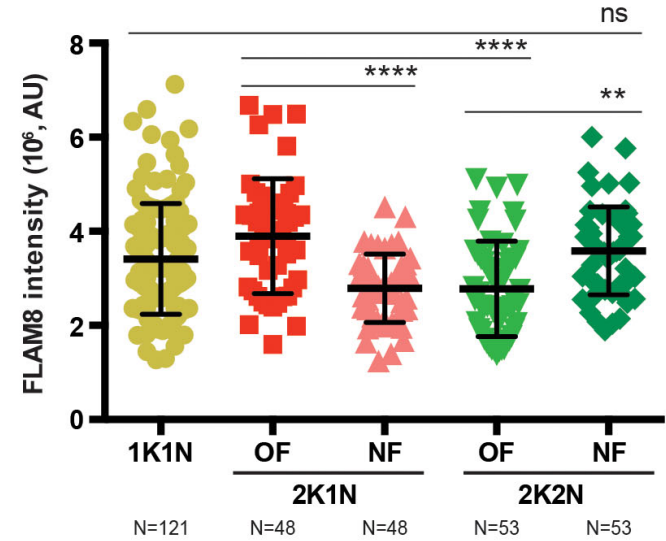

(f)

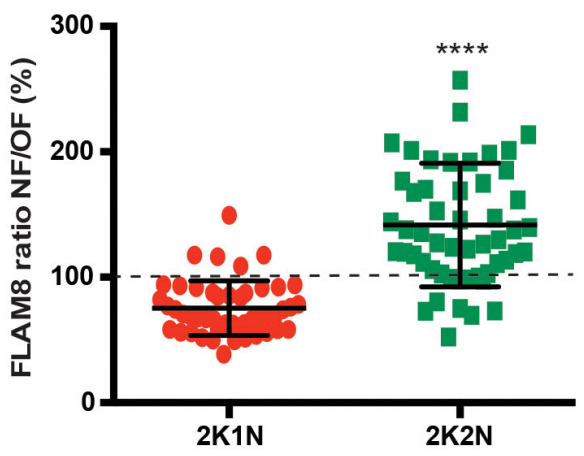

FIGURE 4 FLAM8 accumulates differently in dividing procyclics from posterior versus anterior midgut. Glossina m. morsitans tsetse flies were fed with a blood meal containing pleomorphic AnTat 1.1E ST parasites. The flies were dissected at distinct time points in order to isolate different MG regions and look for PCF trypomastigote stages. $(a-c)$ Infected tsetse flies were dissected at day 3 for PMG region isolation.

(a) Representative immunofluorescence images of methanol-fixed $2 \mathrm{~K} 1 \mathrm{~N}$ (upper panels) and $2 \mathrm{~K} 2 \mathrm{~N}$ (bottom panels) dividing parasites stained with anti-FLAM8 antibody (green), anti-axoneme marker mAb25 (magenta) and DAPI staining (blue). White arrowheads indicate the old and new flagella (OF and NF, respectively). Scale bar is $5 \mu \mathrm{m}$. (b) Quantification of FLAM8 intensity at the flagellum tip of $1 \mathrm{~K} 1 \mathrm{~N}$ parasites and both OF and NF of dividing 2 K1N and 2K2N trypanosomes. Statistically significant differences are indicated with three $(p<.001)$ and four stars $(p<.0001)$. (c) Ratio between FLAM8 intensity in the new flagellum (NF) and the old flagellum (OF) in $2 \mathrm{~K} 1 \mathrm{~N}$ and $2 \mathrm{~K} 2 \mathrm{~N}$ trypanosomes. Statistically significant differences are indicated with four stars $(p<.0001$ ). Black dotted line indicates signal at $100 \%$ ratio. (d-f) Tsetse flies receiving an infective blood meal were dissected after 11 days for anterior midgut (AMG) isolation. (d) Representative immunofluorescence images of methanol-fixed $2 \mathrm{~K} 1 \mathrm{~N}$ (upper panels) and 2K2N (bottom panels) procyclic cells showing anti-FLAM8 (green), mAb25 signals (magenta) and DAPI staining (blue). White arrowheads indicate the old flagellum (OF) and the new flagellum (NF) of dividing trypanosomes. Scale bar is $5 \mu \mathrm{m}$. (e) Dot plot showing FLAM8 signal quantification at the flagellum tip of $1 \mathrm{~K} 1 \mathrm{~N}$ parasites and $2 \mathrm{~K} 1 \mathrm{~N}$ and $2 \mathrm{~K} 2 \mathrm{~N}$ procyclic cells at both mature (OF) and growing flagella (NF). Statistically significant differences are indicated with two $(p<.01)$ and four stars $(p<.0001)$. (f) Ratio between FLAM8 intensity in the new flagellum (NF) and the old flagellum (OF) in $2 \mathrm{~K} 1 \mathrm{~N}$ and $2 \mathrm{~K} 2 \mathrm{~N}$ trypanosomes. Statistically significant differences are indicated with four stars $(p<.0001)$. Black dotted line indicates signal at $100 \%$ ratio. In (b) and (e), the number of parasites used for quantification ( $N$ ) is indicated below the graphs. Statistical analyses include one-way ANOVA followed Tukey-Kramer post-hoc tests for panels (b) and (e), and unpaired $t$-tests for panels $(c)$ and $(f)$. Results are given as means ( \pm standard deviation, $S D$ ) of biometric measurements of cells from three independent experiments and at least three biological replicates. n.s., not significant 
amount of fluorescence was quantified (Figure 4e). Surprisingly, the amount of FLAM8 at the tip of the new flagellum was significantly higher than in the old flagellum in $2 \mathrm{~K} 2 \mathrm{~N}$ trypanosomes (Figure $4 \mathrm{e}$ ). Actually, the amount of FLAM8 in the growing flagellum of $2 \mathrm{~K} 2 \mathrm{~N} \mathrm{PCF}$ corresponded to that in the mature flagellum of a $1 \mathrm{~K} 1 \mathrm{~N}$ PCF from the anterior midgut (Figure 4e). These findings contrast with the opposite profile seen in PCF dividing in the posterior midgut (Figure 4a) and with previous observations in cultured trypanosomes (Bertiaux et al., 2018; Subota et al., 2014). In parallel, the amount of FLAM8 in the old flagellum was significantly decreasing during cell division (Figure 4e, compare red squares with green triangles). Furthermore, significant differences were observed when the ratios of FLAM8 intensities in the NF versus OF of dividing $2 \mathrm{~K} 1 \mathrm{~N}$ and $2 \mathrm{~K} 2 \mathrm{~N}$ were compared in parasites coming from the posterior and the anterior midgut regions (Figure 4c,f, respectively).

Then, we hypothesised that these differences in FLAM8 distribution patterns between dividing PCF in posterior versus anterior midgut could be related to the fate of their respective daughter cells. To test this possibility, FLAM8 localisation profiles were scrutinised in all trypomastigote stages from the midgut and cardia
14 days after ingestion (Figure 5). FLAM8 signal at the distal tip appeared to increase with the parasite development; therefore, the total amount of fluorescence at the tip was subsequently quantified. Since the parasite migratory movement towards the cardia is accompanied by the progression through distinct developmental stages and concomitant with significant changes in flagellar length (Ooi \& Bastin, 2013), this latter parameter was also measured. All trypomastigote parasites were characterised by (a) the length of their flagellum assessed by the axonemal marker mAb25, and (b) their nuclear morphology ranging from a typical rounded shape in PCF from posterior midgut to an elongated conformation in long trypomastigotes (LT) from the anterior midgut and the cardia (Rotureau et al., 2011; Sharma et al., 2008). Interestingly, the amount of FLAM8 at the tip of LT in the cardia was significantly higher than that in PCF from the posterior midgut, and FLAM8 signal appeared more elongated compared to the condensed material found in PCF (Figure 5a,b). Furthermore, the expression of FLAM8 correlated with flagellum length (Figure 5c), increasing during trypomastigote parasite progression into the development from the posterior midgut to the cardia. (a)
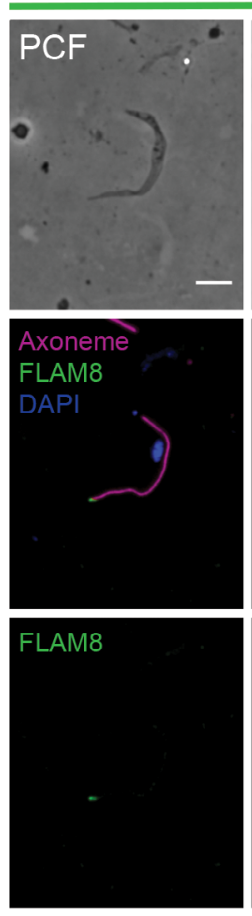

Midgut
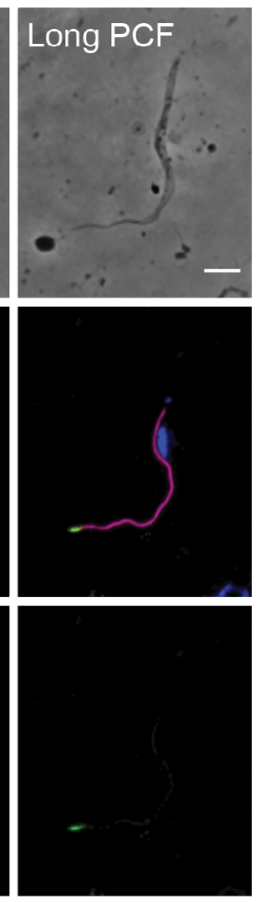

Cardia
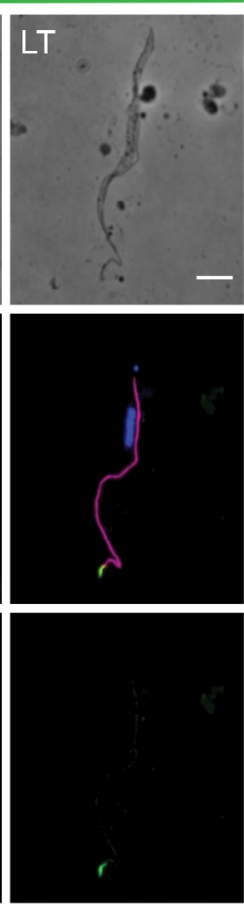
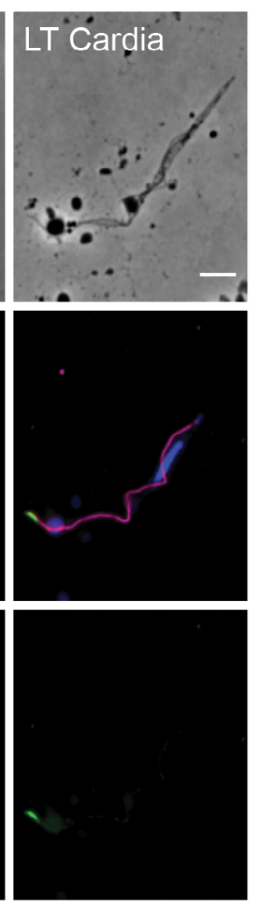

(b)

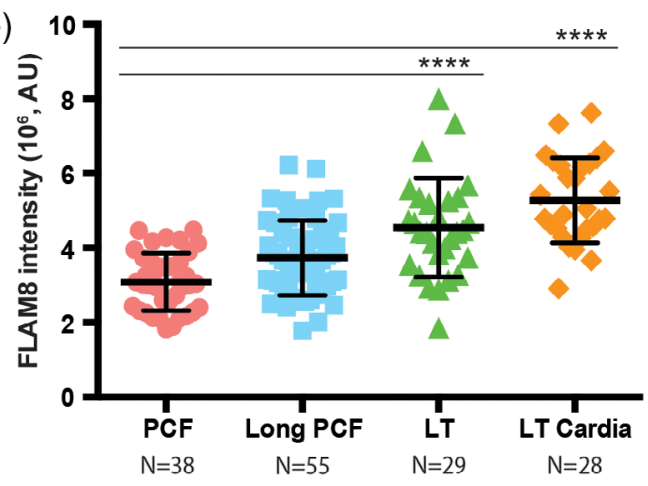

(c)

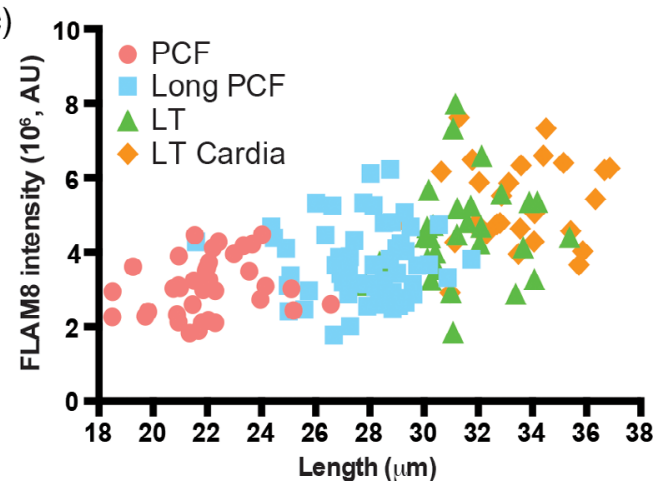

FIGURE 5 FLAM8 distribution at the flagellum tip extends with the elongation of the flagellum in long trypomastigote parasites in the midgut. Tsetse flies were fed with freshly-differentiated AnTat 1.1E stumpy parasites and after 14 days the entire digestive tract including the cardia/proventriculus was isolated for immunofluorescence analyses and further quantification. (a) Selected images of methanol-fixed procyclics, long PCF, LT and LT from the cardia stained with mAb25 axonemal marker (magenta), anti-FLAM8 antibody (green) and DAPI for DNA content (blue). Upper line: midgut stages (green) versus trypomastigotes found in the cardia (red). (b) Dot plot showing the FLAM8 intensity at the tip of procyclic parasites, long PCF and long trypomastigotes (LT) recovered from the midgut and the cardia. Statistically significant differences upon one-way ANOVA followed by Tukey-Kramer post-test are indicated with four stars $(p<.0001)$. The number of parasites used for quantification $(\mathrm{N})$ is indicated below the graph. (c) The mAb25 axonemal signal was used for flagellum length quantification and further correlation with the FLAM8 amount at the tip of specific parasite stages. Results are given as means ( \pm standard deviation, $S D$ ) of biometric measurements of cells from two independent experiments and at least three biological replicates 


\section{5 | FLAM8 distribution along the trypanosome flagellum is stage-specific}

In the tsetse cardia, long dividing epimastigote trypanosomes maintained a similar FLAM8 localisation (Figure 6a). These cells divide asymmetrically to produce long and short epimastigote parasites (Sharma et al., 2008; Van den Abbeele et al., 1999). While long epimastigotes showed a FLAM8 localisation concentrated at the tip, short epimastigote cells (the shortest morphological form identified with a flagellum length about 3-4 $\mu \mathrm{m}$ ), exhibited a distinct distribution of FLAM8 along the entire flagellum length (Figure 6a). Indeed, short epimastigote trypanosomes are assumed to be transported by the highly motile long epimastigotes into the lumen of the salivary gland, where they will attach to the epithelium and differentiate into attached epimastigote forms. The pattern observed in short epimastigotes was also observed in salivary gland parasites, that is, in (a)
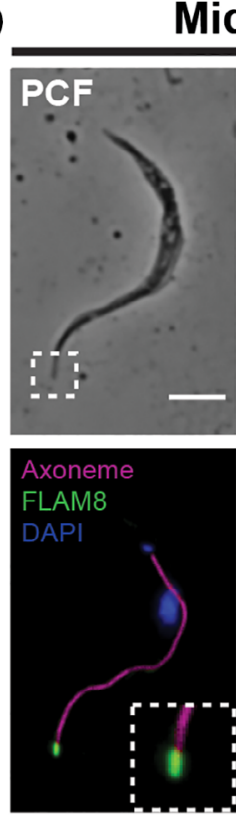

(b)
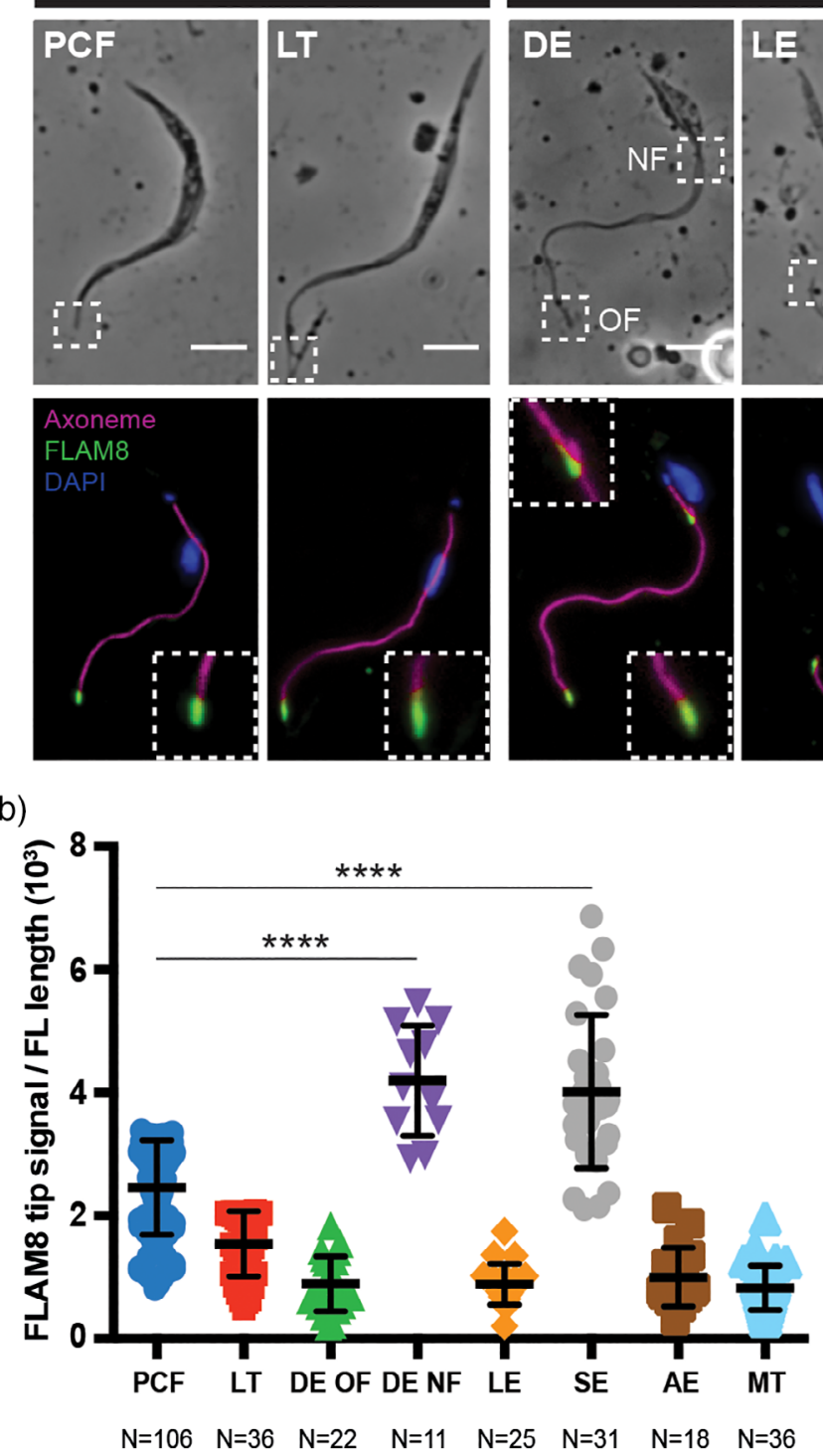

$\mathrm{N}=106 \mathrm{~N}=36 \quad \mathrm{~N}=22 \quad \mathrm{~N}=11 \quad \mathrm{~N}=25 \quad \mathrm{~N}=31 \quad \mathrm{~N}=18 \quad \mathrm{~N}=36$
Cardia and foregut

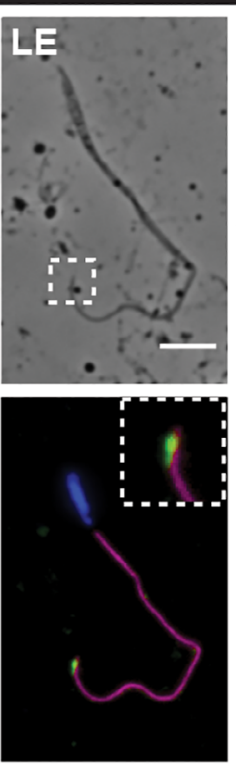

(c)
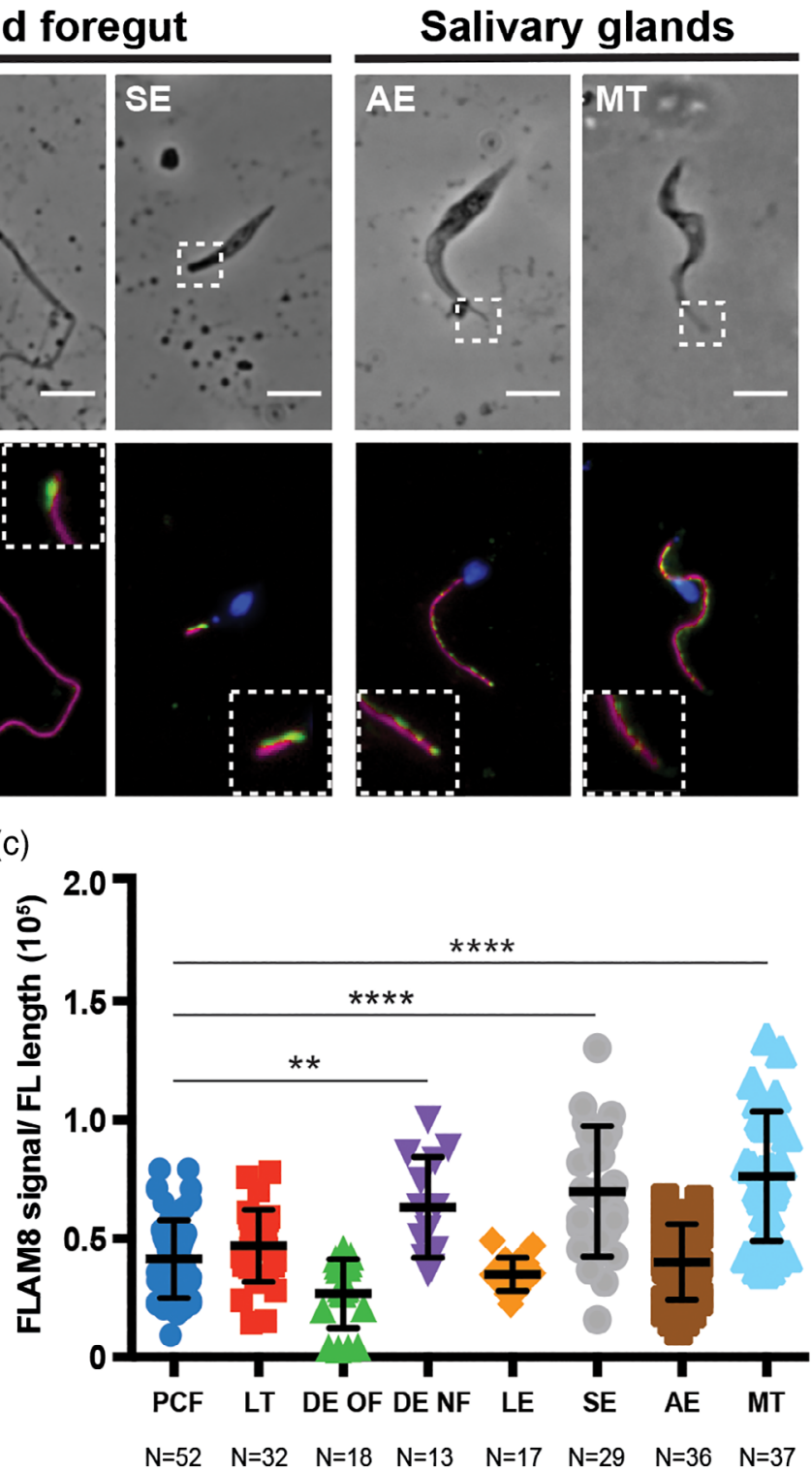

FIGURE 6 FLAM8 is redistributed along the trypanosome flagellum twice during the parasite cycle in the insect vector. (a) AnTat 1.1E stumpy parasites were used to infect Glossina m. morsitans tsetse flies in order to collect fly tissues (midgut, cardia and salivary glands), and look for all developmental stages after 30 days of infection. Immunofluorescence images using anti-FLAM8 antibody (green), anti-axonemal marker mAb25 (magenta) and DAPI staining (blue). White dashed-line boxes show a two-fold magnification of the distal tip in all parasite stages. Scale bar is $5 \mu \mathrm{m}$. (b) and (c) The ratio of intensity of FLAM8 fluorescence at the distal tip (b) and along the entire flagellum (c) related to flagellum length was quantified in all trypanosome morphotypes found within the midgut, cardia and the salivary glands of infected tsetse flies. The number of cells used for the quantification $(\mathrm{N})$ is indicated below the graphs. Statistically significant differences after one-way ANOVA followed by Tukey-Kramer post-tests are indicated with two $(p<.01)$ and four stars $(p<.0001)$. Results are given as means ( \pm standard deviation, SD) of biometric measurements of cells from three independent experiments and at least three biological replicates. AE, attached epimastigote; $D E$, dividing epimastigote; LE, long epimastigote; LT, long trypomastigote; MT, metacyclic; PCF, procyclic; SE, short epimastigote 
both attached epimastigote trypanosomes and infective metacyclic trypomastigote cells (Figure 6a). A quantitative analysis of the fluorescence was performed in all stages by calculating the ratio between the integrated intensity of the FLAM8 signal in the flagellum and the flagellum length (Figure $6 \mathrm{~b}, \mathrm{c}$ ), and two intensity analyses were performed: one based on the FLAM8 signal found at the distal tip by drawing an region of interest with a fixed size for all parasite stages (Figure $6 \mathrm{~b}$ ), and one along the entire flagellum length by using the mAb25 signal labelling the axoneme (Figure 6c). In both quantitative analyses, the shortest flagellum corresponding to the new flagellum of the dividing epimastigotes and the flagellum of the short epimastigotes presented the highest ratios, compatible with the redistribution/dilution of FLAM8 to the entire length of the flagellum as early as in short epimastigote parasites from the cardia.

Then, in the salivary glands, a continuous production of infective metacyclic parasites is ensured through an asymmetric division that results in two distinct daughter cells, one inheriting the old flagellum that displays the epimastigote configuration and the one inheriting the new flagellum, which adopts the trypomastigote configuration (Rotureau et al., 2012). In order to investigate in more depth the dynamics of FLAM8 redistribution in salivary gland parasites undergoing metacyclogenesis, transition stages between epimastigote and trypomastigote, that is, 'Epi-Trypo' dividing cells, were analysed (Figure 7). Interestingly, during the asymmetric division in $2 \mathrm{~K} 1 \mathrm{~N}$ 'EpiTrypo' cells, a higher amount of FLAM8 was found along the growing new flagellum (Figure 7a,b), a significant enrichment that was maintained in pre-metacyclic cells and even more pronounced in infective metacyclic trypomastigotes (Figure 7a,b).

\section{3 | DISCUSSION}

\subsection{FLAM8 and the flagellum tip}

FLAM8 was first detected at the distal tip of PCF trypanosomes in culture (Subota et al., 2014), an observation that has been confirmed in insect-derived PCF parasites in the present study. Only a small subset of flagellar proteins have also been exclusively localised to the distal end of the flagellum in PCF, suggesting the existence of a very specific microdomain (Chan \& Ersfeld, 2010; Liu et al., 2010; Saada et al., 2014; Varga et al., 2017; Woodward et al., 1995). The repetition of PFAMB506 domains (Pfam ID PB000506), also found in 4 T. brucei flagellum attachment zone proteins as well as in a few other cytoskeletal associated proteins (Sunter et al., 2015), could be involved in the interactions of FLAM8 with cytoskeletal partners at the flagellum tip, albeit the function of this domain remains unknown. However, FLAM8 was not detected by immunoprecipitation in PCF flagellar tip extracts (Varga et al., 2017). As proposed in Chlamydomonas where HSP70 and EB1 have been localised to the distal tips of the flagella (Bloch \& Johnson, 1995; Pedersen, Geimer, \& Rosenbaum, 2006), FLAM8 was speculated to be involved in microtubule assembly, stability and/or dynamics at the distal tip of the axoneme. In addition, the (a)

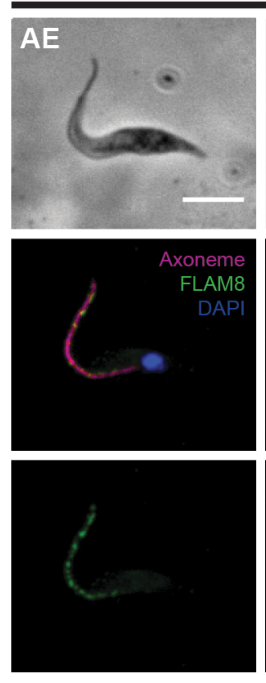

Metacyclogenesis

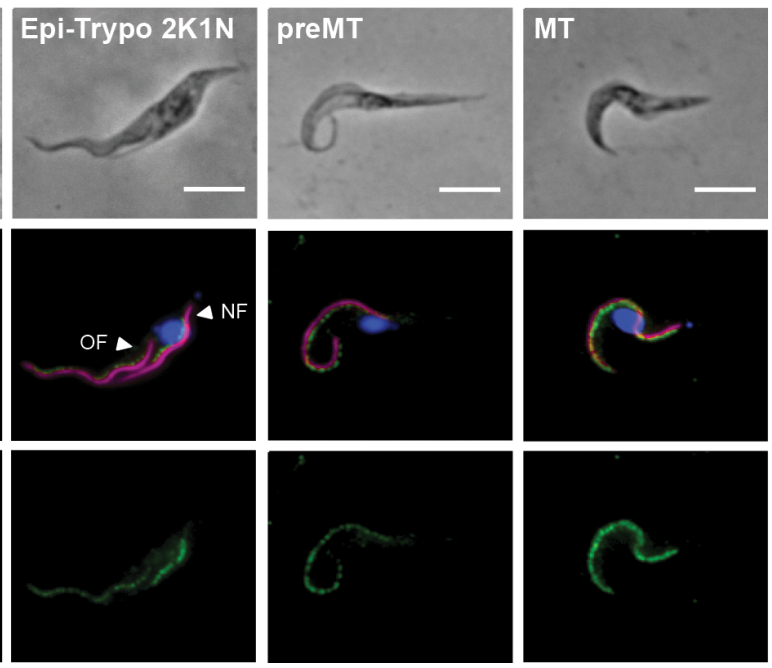

(b)

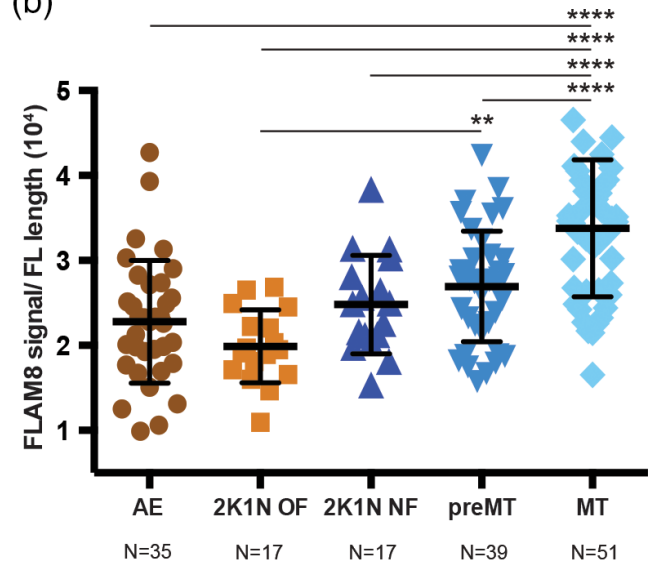

FIGURE 7 FLAM8 is differentially distributed during the asymmetric division in the salivary glands. (a) Representative images of 'Epi-Trypo' transition in the salivary glands. Methanol-fixed AnTat 1.1E parasites were stained with anti-FLAM8 antibody (green), anti-axonemal marker mAb25 (magenta) and DAPI staining (blue). White arrowheads within the 'Epi-Trypo' panel indicate old (OF) and new (NF) flagellum. Scale bar is $5 \mu \mathrm{m}$. (b) Quantification of FLAM8 fluorescence intensity along the flagellum related to the length of the axoneme. The number of parasites considered for quantification $(\mathrm{N})$ is indicated below the graphs. Statistically significant differences after one-way ANOVA followed by TukeyKramer post-test are indicated with two $(p<.01)$ and four stars $(p<.0001)$. Results are given as means $( \pm$ standard deviation, SD) of biometric measurements of cells from four independent experiments and at least three biological replicates. 2K1N, 'Epi-Trypo' parasite during the asymmetric division; AE, attached epimastigote; MT, metacyclic; preMT, pre-metacyclic 
redistribution of FLAM8 along the entire flagellum in BSF indicates that FLAM8 may not exclusively and permanently reside within such a distal tip location.

FLAM8 amount increases during flagellum construction and after cell division in PCF in culture (Subota et al., 2014), and it is maintained at the distal tip of the flagellum by IFT activity (Fort et al., 2016). FLAM8 could be part of a stable structure that accumulates material over time or could be turning over and be actively maintained at the distal tip, for example, by the action of IFT. This was addressed in (Subota et al., 2014) by performing FRAP (fluorescence recovery after photobleaching) analysis in cells bearing a single flagellum. Two rather different situations were encountered that could be explained by the different age of the flagella: in half of the cells presumably bearing a mature flagellum, recovery was either limited or even barely detectable, whereas in the remaining cells with an elongating or immature flagellum, significant recovery was observed, indicating a relatively rapid turnover compatible with IFT. Here, in both dividing BSF and PCF isolated from infected blood and tsetse midguts, respectively, we confirmed that FLAM8 is progressively added to the tip of the new flagellum during its assembly. Other flagellar tip proteins display a similar pattern: the Axonemal Capping protein (ACS1), whose amount in the new flagellum is length-dependent in PCF (Varga et al., 2017), and two insect stagespecific adenylate cyclases, ACP1 and ACP4 (Saada et al., 2014). FLAM8 proteins newly synthesised in the cytoplasm need to transit through the transition zone that acts as a gate between the flagellum and the cell body compartments (Reiter, Blacque, \& Leroux, 2012). Although incorporation of FLAM8 in the new flagellum in dividing PCFs in the posterior midgut is likely to result from de novo synthesis of fresh FLAM8 material brought from the base to the tip by IFT, it could conceivably be different in the case of dividing PCFs in the anterior midgut. Possibly in addition to de novo synthesis, the concomitant FLAM8 amount increase in the new flagellum and decrease in the old one during cell division in PCFs from anterior midgut could also result, at least partially, from a recycling of FLAM8 from the mature flagellum to the new flagellum. Some of the FLAM8 proteins present at the tip of the old flagellum could be transferred to the new flagellum by IFT successively via the IFT retrograde machinery in the old flagellum and the IFT anterograde machinery of the new flagellum, via the flagellar bases in the cytoplasm, although an exchange between the two flagella has not been proven so far. A variation in the number of FLAM 8 binding sites at the tip of the old flagellum, with a free circulation of FLAM8 between the two flagella could also be envisaged.

\subsection{FLAM8 as a flagellum maturation marker}

Recently, the grow-and-lock model was proposed to explain how flagellum length could be regulated in procyclic trypanosomes in culture (Bertiaux et al., 2018). FLAM8 was identified as a marker of the locking event associated to the acquisition of the final flagellum length. Here, FLAM8 was also seen to progressively accumulate at the tip during the entire flagellum elongation process in SL BSF and the inhibition of cell division resulted in the production of BSF parasites bearing two long flagella with equal amount of FLAM8 at the distal tip, in contrast to dividing cells where FLAM8 signal in the new flagellum only reaches $80 \%$ of the amount present in the old one. These observations suggest that the locking event, whose exact molecular nature needs to be elucidated, is controlled by a general cellular process that is temporally regulated, and could possibly be involved in discriminating the progression through another cell cycle or a new round of differentiation.

In line with the proposal that PCF division results in the production of two daughter cells that are not equivalent (Abeywickrema et al., 2019), we reasoned that the discrepancy between the two FLAM8 distribution patterns observed between the two flagella of early dividing PCF from the posterior midgut (PMG) and late dividing PCF from the anterior midgut could somehow reflect the distinct fate of their respective daughter cells bearing the new flagellum (Figure 8). Early dividing PCF would keep on proliferating at a regular rate by producing daughter cells destined to further re-enter new cell division cycles in the PMG. In this context, FLAM8 is detected in lower amount in the new flagellum than in the old flagellum in $2 \mathrm{~K} 2 \mathrm{~N}$ early PCF, resulting in the production of a daughter cell with a slightly shorter flagellum that would further mature and elongate after cytokinesis, up to the same length as that of the old flagellum (Figure 8), as proposed in the grow-and-lock model (Bertiaux et al., 2018). By contrast, part of the PCF population is assumed to elongate and to migrate to the tsetse cardia before differentiating into long asymetrically dividing epimastigotes (Lemos et al., 2020; Rotureau \& Van Den Abbeele, 2013). Interestingly, we found that FLAM8 amount at the flagellum tip increases with the elongation of the flagellum during trypomastigote parasite progression from the PMG towards the anterior region and, finally, in the cardia. A second population of dividing PCF that would first migrate towards the anterior midgut and start dividing in a different manner in order to produce the future LT that would further enter differentiation into the next stage was further characterised. In this case, late PCF found to divide in the anterior midgut would do so in an asymmetrical manner, as assessed by the peculiar FLAM8 distribution in the two flagella: FLAM8 is detected in higher amounts in the new flagellum than in the old flagellum in $2 \mathrm{~K} 2 \mathrm{~N}$ late $\mathrm{PCF}$, resulting in the production of a daughter cell with a flagellum that would further mature and elongate after cytokinesis (Figure 8). This situation could be compared to the differential distribution of calflagins in 'Epi-Trypo' asymmetrically dividing cells in the salivary glands, resulting in the production of two distinct cells with different fates (Rotureau et al., 2012). Thus, we propose that the amount of FLAM8 at the tip of the new flagellum of $2 \mathrm{~K} 2 \mathrm{~N}$ PCF may represent a potential marker of prediction for the future of the daughter cell that could become either a proliferative PCF in the PMG or a long trypomastigote about to differentiate in epimastigote in the anterior midgut (Figure 8).

\section{3 | Differential distribution of FLAM8 during the parasite cycle}

The model presented in Figure 8 recapitulates all our observations and highlights that FLAM8 amount and distribution along the trypanosome flagellum are dynamic and stage-specific. A number of stage- 


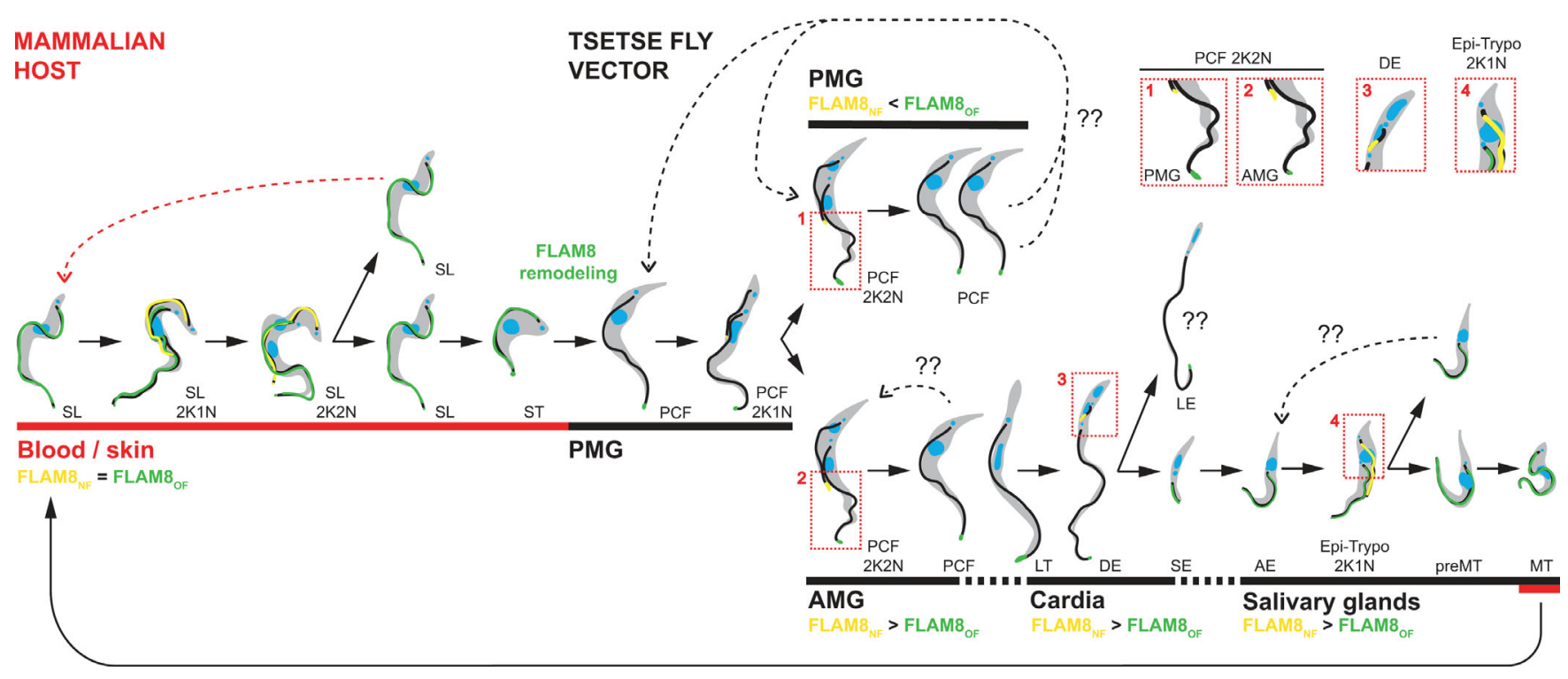

FIGURE 8 FLAM8 is a marker of the flagellum maturation state that predicts the fate of a trypanosome during the parasite cycle. In the mammalian host, proliferative slender (SL) parasites divide in order to colonise the intravascular and extravascular compartments. This symmetric division gives rise to one daughter cell that will undergo another round of division or that will stop proliferation and differentiate into arrested stumpy trypanosomes (ST). Both SL and ST trypomastigotes exhibit FLAM8 along the full flagellum length. During a blood meal on an infected host, a tsetse fly will ingest a pool of SL and ST cells, but only the latter will be capable of surviving in the new host environment. In the PMG, ST will rapidly transform into proliferative procyclic (PCF) trypomastigotes. This event will be characterised by the dramatic remodelling of FLAM8 distribution in the existing flagellum, since PCF cells display a FLAM8 concentrated only at the very distal tip of the flagellum. In addition, $2 \mathrm{~K} 2 \mathrm{~N}$ PCF cells dividing in the PMG express a lower amount of FLAM8 in the growing flagella, associated with a post-division maturation of the NF in the daughter cell according to the grow-and-lock model. The daughter cell would then probably re-enter another proliferation cycle in order to maintain the proliferative PCF population in the PMG. In contrast, dividing procyclic cells located in the anterior midgut present a higher amount of FLAM8 in the NF before cytokinesis, suggesting that the daughter cell would proceed to the next developmental step and differentiate into non-proliferative long trypomastigotes (LT) by a drastic elongation of their flagella. These elongated trypomastigote parasites will subsequently enter the cardia in order to initiate a differentiation into epimastigote stages. During the asymmetric division performed by these long dividing epimastigotes (DE), two daughter cells are generated: a long epimastigote (LE), which is believed to die shortly after migration and/or cytokinesis and a short epimastigote (SE) that will further develop in the salivary glands. Unlike DE and LE, SE present FLAM8 along the entire length of the flagellum, evidencing a second redistribution of the protein in vivo. Once in the salivary glands, SE will differentiate into stages that will attach to the salivary epithelium, keeping FLAM8 along their entire flagellum. Interestingly, during the asymmetric division carried out by 'Epi-Trypo' dividing $A E$, the daughter cell inheriting the NF exhibits a higher amount of FLAM8 as compared to the OF. This daughter cell will then differentiate into pre-metacyclic (preMT) parasites and, finally, into infective metacyclic trypomastigotes, with FLAM 8 being present along the entire flagellum length. Red dotted boxes show the magnification of particular trypanosome areas indicated with numbers. Colour-code: the axoneme is depicted in black while the FLAM8 distribution is shown in green in the OF and in yellow in the NF. FLAM8 $8_{\mathrm{NF}}$, FLAM8 amount in the new flagellum; FLAM8 $8_{\mathrm{OF}}$, FLAM8 amount in the old flagellum; K, kinetoplast; $\mathrm{N}$, nucleus

specific proteins are well-characterised in African trypanosomes, especially surface antigens such as Variant Surface Glycoproteins, procyclin (Roditi, Carrington, \& Turner, 1987) or Brucei Alanine-Rich Protein (Urwyler, Studer, Renggli, \& Roditi, 2007), but only a few stage-specific markers are known to be restricted to the flagellum. Members of the calflagin family are expressed during metacyclogenesis in attached epimastigote parasites asymmetrically dividing in the tsetse salivary glands. These membrane-associated proteins are progressively enriched exclusively in the new flagellum of the trypomastigote daughter cell that will further mature into an infective metacyclic form in the saliva (Rotureau et al., 2012). By contrast, here, by ex vivo differentiation of BSF into PCF parasites, we demonstrated that FLAM8 redistribution takes place in a few hours in $1 \mathrm{~K} 1 \mathrm{~N}$ cells with a procyclic morphology. To our knowledge, this is the first evidence of a molecular remodelling occurring in the existing flagellum of a non-dividing T. brucei cell. Another type of remodelling consisting in a flagellum shortening has recently been described in $1 K 1 \mathrm{~N} T$. congolense parasites from the tsetse cardia (Peacock, Kay, Bailey, \& Gibson, 2018). These observations raise a number of questions regarding the mechanisms involved and their impact on the flagellum length control. A quantitative phosphoproteomic comparison of BSF and PCF trypanosomes revealed differential protein phosphorylation levels among flagellar and RNA-binding proteins that are crucial for parasite differentiation (Urbaniak, Martin, \& Ferguson, 2013). This is the case of FLAM8 that presents differential phosphorylation between the two stages with four sites phosphorylated exclusively in PCF and a distinct one in BSF. This could be linked to its differential distribution in the two stages, hence possibly to distinct functions. 
FLAM8 is then found concentrated at the tip of all trypomastigote stages in the midgut in amounts directly related to their flagellum length. However, it is detected along with the entire length of the flagellum in short epimastigote cells (Figure 8). This change of FLAM8 profile takes place during the construction of the new short flagellum in long asymmetrically dividing epimastigotes. At this step also, FLAM8 could be involved in flagellum length control, with a higher amount of FLAM8 being already produced in short epimastigotes to anticipate the upcoming rapid flagellum elongation necessary for a proper attachment to the salivary gland microvilli. Recent evidence has proved that trypanosome stages developing in the insect vector possess a total amount of IFT proteins that directly correlates with the length of the flagellum (Bertiaux et al., 2018). Interestingly, short epimastigotes were found as the sole parasite stage to exhibit a supplementary large amount of dynamic IFT material at the flagellar distal end (Bertiaux et al., 2018). In the salivary gland stages, FLAM8 is also a marker of the flagellum maturation state that appears to predict the fate of attached 'Epi-Trypo' dividing cells, with an enrichment of FLAM8 in the flagellum in the trypomastigote configuration as compared to the epimastigote one (Figure 8). This situation could also be compared to the differential distribution of calflagins in 'Epi-Trypo' asymmetrically dividing cells (Rotureau et al., 2012).

In summary, we propose that FLAM8 could be considered a meta-marker of the flagellum stage and maturation state in trypanosomes. FLAM8 distribution in trypanosomes is stage-specific and its remodelling seems to occur in two different situations: (a) in a new flagellum produced during cell divisions in PCF of the anterior midgut, in long epimastigotes of the cardia and in 'Epi-Trypo' attached to the salivary glands, (b) or in the existing flagellum of non-dividing ST BSF differentiating into PCF in the midgut. Noteworthily, this drastic remodelling of FLAM8 distribution in an existing flagellum is concomitant to major environmental changes for the parasites. Further research is needed to fully understand the roles of FLAM 8 in the tsetse insect vector. In addition, understanding the potential protein interactions with other flagellum partners, especially at the distal tip, is another challenge that would help to unravel the essential roles of this fascinating and essential organelle.

\section{4 | EXPERIMENTAL PROCEDURES}

\subsection{Strains, culture and in vitro differentiations}

The AnTat 1.1E Paris pleomorphic clone of $T$. brucei brucei was derived from a strain originally isolated from a bushbuck in Uganda in 1966 (Le Ray, Barry, Easton, \& Vickerman, 1977). Procyclic form trypanosomes (PCF) were grown at $27^{\circ} \mathrm{C}$ in SDM-79 medium (Brun \& Schonenberger, 1979) supplemented with $10 \%(\mathrm{v} / \mathrm{v})$ heat-inactivated foetal bovine serum (FBS), haemin $(7.5 \mathrm{mg} / \mathrm{ml})$ and $8 \mathrm{mM}$ glycerol (SDMG). Bloodstream form trypanosomes (BSF) of the same strain were cultivated in HMI-11 medium supplemented with $10 \%(\mathrm{v} / \mathrm{v})$ FBS
(Hirumi \& Hirumi, 1989) at $37^{\circ} \mathrm{C}$ in $5 \% \mathrm{CO}_{2}$. For in vitro slender to stumpy BSF differentiation, we used 8-pCPT-2'-O-Me-5'-AMP, a nucleotide analogue of $5^{\prime}$-AMP from BIOLOG Life Science Institute (Germany). Briefly, $2 \times 10^{6}$ pleomorphic AnTat $1.1 \mathrm{E}$ slender forms were incubated with 8-pCPT-2'-O-Me-5'-AMP $(5 \mu \mathrm{M})$ for $48 \mathrm{hr}$ (Barquilla et al., 2012). Freshly differentiated stumpy form parasites were then centrifuged at $1,400 \mathrm{~g}$ for $10 \mathrm{~min}$ in TDB (Trypanosome Dilution Buffer: $5 \mathrm{mM} \mathrm{KCl}, 80 \mathrm{mM} \mathrm{NaCl}, 1 \mathrm{mM} \mathrm{MgSO}{ }_{4} \cdot 7 \mathrm{H}_{2} \mathrm{O}$, $20 \mathrm{mM} \mathrm{Na}_{2} \mathrm{HPO}_{4}, 2 \mathrm{mM} \mathrm{NaH}_{2} \mathrm{PO}_{4}, 20 \mathrm{mM}$ glucose) and resuspended in SDMG medium with $10 \mathrm{mM}$ glutathione for tsetse fly infection (MacLeod, Maudlin, Darby, \& Welburn, 2007). For in vitro differentiation into PCF, freshly differentiated short stumpy form trypanosomes were transferred into SDM-79 medium supplemented with $10 \%(\mathrm{v} / \mathrm{v})$ FBS, $6 \mathrm{mM}$ cis-aconitate and $20 \mathrm{mM}$ glycerol at $27^{\circ} \mathrm{C}$ (Czichos, Nonnengaesser, \& Overath, 1986).

\section{2 | Generation of cell lines expressing fluorescent FLAM8}

For EYFP tagging of FLAM8 at the C- and N-terminal ends, p3329 and p2675 plasmids were used, respectively (Kelly et al., 2007). These vectors carry different FLAM 8 gene fragments, corresponding to FLAM8 ORF nucleotides 8892-9391 and 4-489 in the p3329 and p2675 plasmids, respectively. Both vectors were linearised with Nrul and BamHI, respectively, prior to nucleofection following a standard protocol (Burkard et al., 2007). Transformants were selected with appropriate puromycin concentrations (1 or $0.1 \mu \mathrm{g} / \mathrm{ml}$ for PCF or BSF). Clonal populations were obtained by limiting dilution. Cell culture growth was monitored with an automatic Muse cell analyser (Merck Millipore, Paris). Neither the FLAM8 targeting to the tip nor the flagellum length was affected by the presence of any EYFP-tags in PCF cells (Figure S1a,b).

\section{3 | Tsetse fly maintenance, infection and dissection}

Glossina morsitans morsitans tsetse flies were maintained in Roubaud cages at $27^{\circ} \mathrm{C}$ and $70 \%$ hygrometry and fed through a silicone membrane with fresh mechanically defibrinated sheep blood. Teneral males (between 24 and $72 \mathrm{hr}$ post-emergence) were allowed to ingest parasites in culture medium during their first meal through a silicone membrane. Here, we used fresh in vitro differentiated stumpy BSF trypanosomes resuspended at $2 \times 10^{6}$ cells $/ \mathrm{ml}$ in complete SDM-79 medium supplemented with $10 \mathrm{mM}$ glutathione prior to infection (MacLeod et al., 2007).

Flies were starved for at least $24 \mathrm{hr}$ before being dissected. Different time points were selected for fly dissection: (a) 3 days for early isolation of PCF from the PMG region; (b) 11 days for recovery of PCF and LT from the anterior midgut (AMG); (c) 14 days for isolation of all trypomastigote parasites from the midgut and cardia and, (d) 30 days for isolation of all stages from the MG, PV and salivary 
glands (SG). For posterior and anterior midgut isolation, the entire midgut was cut into three subsections, including the PMG close to the hindgut, the middle part of the midgut until the appearance of the bacteriome and the thin anterior midgut that runs from the cardia to the bacteriome. They were isolated accordingly and placed in a drop of phosphate buffer saline (PBS). For recovery of all tsetse organs, after rapid isolation of the salivary glands in a first drop of PBS, whole tsetse alimentary tracts, from the distal part of the foregut to the Malpighian tubules, were dissected and arranged lengthways in another drop of PBS as previously described (Rotureau et al., 2011, 2012). Isolated organs were then either scrutinised under a microscope at $40 \times$ magnification and imaged, or individually dilacerated, dried on the slide and further treated for immuno-fluorescence (Rotureau et al., 2014).

\section{4 | Mice infection and ethical statements}

Seven-week old male BALB/c mice were purchased from Janvier Laboratory (substrain BALB/cAnNRj) and used as models for experimental infection. This study was conducted in strict accordance with the recommendations from the Guide for the Care and Use of Laboratory Animals of the European Union (European Directive 2010/63/UE) and the French Government. The protocol was approved by the 'Comité d'éthique en expérimentation animale de l'Institut Pasteur' CETEA 89 (Permit number: 2012-0043 and 2016-0017) and undertaken in compliance with the Institut Pasteur Biosafety Committee (protocol CHSCT 12.131). BR is authorised to perform experiments on vertebrate animals (licence \#A-75-2035) and is responsible for all the experiments conducted personally or under his supervision. For in vivo infections, two BALB/c mice were injected intraperitoneally (IP) with $10^{5} \mathrm{SL}$ BSF parasites, washed in TDB and resuspended in $100 \mu \mathrm{l}$ PBS prior animal inoculation. Parasitemia was determined daily from tail bleeds. At the first peak of parasitemia, blood containing a mixture of SL and ST parasites was sampled and used to induce the ex vivo differentiation of ST parasites into PCF trypanosomes.

\section{5 | Immuno-fluorescence analysis}

Cultured parasites were washed twice in SDM-79 medium without serum or in TDB for PCF and BSF, respectively, and spread directly onto poly-L-lysine coated slides. Both methanol, paraformaldehyde/ methanol and cytoskeleton fixation protocols were used for IFA. For methanol fixation, slides were air-dried for $10 \mathrm{~min}$, fixed in methanol at $-20^{\circ} \mathrm{C}$ for $5 \mathrm{~min}$ and rehydrated for $20 \mathrm{~min}$ in PBS. For $\mathrm{PFA} / \mathrm{methanol}$, spread cells were left for $10 \mathrm{~min}$ to settle prior to treatment with 1 volume 4\% PFA solution in PBS at $\mathrm{pH}$ 7. After 5 min, adherent PFA-treated cells were washed briefly in PBS before being fixed with methanol at $-20^{\circ} \mathrm{C}$ for an additional 5 min followed by a rehydration step in PBS for 15 min. For IFA on cytoskeletons, $40 \mu \mathrm{l}$ of TDB washed cells were spread on a poly-lysine slide. After sedimentation in humid atmosphere, cells were treated for 5 min by addition of $70 \mu \mathrm{l}$ of $0,4 \%$ Nonidet P-40 (IGEPAL CA-630, Fluka) in PIPES buffer (100 mM PIPES pH 6.9 adjusted with $\mathrm{NaOH}, 1 \mathrm{mM}$ $\mathrm{MgCl}_{2}$ ), then washed twice in PIPES buffer and fixed at $-20^{\circ} \mathrm{C}$ in methanol for $5 \mathrm{~min}$. Cells were rehydrated in PBS for $15 \mathrm{~min}$ prior to immunodetection by IFA.

For immunodetection, slides were incubated for $1 \mathrm{hr}$ at $37^{\circ} \mathrm{C}$ with the appropriate dilution of the first antibody in 0.1\% BSA in PBS. After three consecutive 5 min washes in PBS, species and subclassspecific secondary antibodies coupled to the appropriate fluorochrome (Alexa 488, Cy3, Cy5 Jackson ImmunoResearch) were diluted $1 / 400$ in PBS containing $0.1 \%$ BSA and were applied for $1 \mathrm{hr}$ at $37^{\circ} \mathrm{C}$. After washing in PBS as indicated above, slides were finally stained with 4',6-diamidino-2-phenylindole (DAPI, $1 \mu \mathrm{g} / \mathrm{ml}$ ) for visualisation of kinetoplast and nuclear DNA content, and mounted under coverslips with ProLong antifade reagent (Invitrogen), as previously described (Rotureau et al., 2011).

Slides were observed under an epifluorescence DM14000 microscope (Leica) with a 100× objective (NA 1.4), an EL6000 (Leica) as light excitation source and controlled by the Micro-Manager V1.4.22 software (NIH) and images were acquired using a Hamamatsu ORCA03G or Prime 95B (Photometrics) cameras. Images were analysed with ImageJ V1.8.0 (NIH). For each comparative analysis, all samples were acquired using identical exposure times and all images are displayed using the same settings.

Structured illumination microscopy was performed on a Zeiss LSM 780 Elyra PS1 microscope (Carl Zeiss, Germany) using PlanApochromat $63 \AA$ Å /1.4 oil objective with a 1.518 refractive index oil (Carl Zeiss). The fluorescence signal was detected on an EMCCD Andor Ixon $8871 \mathrm{~K}$. SIM images were processed with the ZEN software and corrected for chromatic aberration using 100-nm TetraSpeck microspheres (ThermoFisher ScienUfic). The SIM check plugin in ImageJ was used to analyse the quality of the acquisition and the processing in order to optimise parameters for resolution, signalto-noise ratio and reconstruction pattern.

Primary antibodies were: mAb25 (anti-mouse IgG2a, 1:10), a monoclonal antibody used as a flagellum marker as it specifically recognises the axoneme protein TbSAXO1 (Dacheux et al., 2012); L8C4 (anti-mouse IgG1, 1:10; Kohl et al., 1999), a monoclonal antibody that recognises the PFR2 protein and served as a PFR marker; an anti-GFP rabbit serum (Invitrogen \#A6455, 1:300) to label the EYFP-tagged versions of FLAM8; a rabbit anti-FTZC (1:1,000), which recognises the FTZC protein in the transition zone (Bringaud et al., 2000); antiAK antiserum against $T$. cruzi arginine kinase (anti-mouse IgG, 1:500) (Subota et al., 2014). FLAM8 was detected using anti-FLAM8 rabbit serum (1:500). Specifically, to generate the polyclonal anti-FLAM8 antibody, rabbits were immunised with His-tagged recombinant protein (encoding a 151 amino acid sequence repeated twice in the FLAM8 protein; corresponding to amino acids 442-592 and 593-743). Recombinant protein was expressed in E. coli and purified to homogeneity using immobilised metal affinity chromatography (IMAC) and anion exchange chromatography. Stumpy BSF and early PCF stages 
were identified at the molecular level with a rabbit polyclonal antiPAD1 antibody (kindly provided by Keith Matthews, University of Edinburgh; dilution 1:300) and an anti-EP procyclin (Cedarlane ${ }^{\circledR}$, Canada; clone TBRP1/247 anti-mouse IgG1, 1:500), respectively. When required, sequential incubation with antibodies was performed by means of a saturation step containing 1\% BSA in PBS.

\section{6 | Measurements, normalisation and statistical analyses}

Normalisation of fluorescent signals was carried out by the parallel adjustments of the raw integrated density and/or minimum signal and/or maximum signal in all the images to be compared in ImageJ V1.8.0 (NIH). For flagellum length measurements, the mAb25 staining of the axoneme was taken as reference using ImageJ. For clarity purposes, the brightness and contrast of several pictures were adjusted after their analysis in accordance with editorial policies. Statistical analyses and plots were performed with XLSTAT 2019.2.01 (Addinsoft) in Excel 2016 (Microsoft) or Prism V8.2.1 (GraphPad). Statistical analyses include linear regression followed by $t$-tests for twogroup comparisons and one-way ANOVA followed by Tukey-Kramer post-tests when more than two groups were present, always at $95 \%$ confidence. The number of samples analysed for each experiment is indicated in figure legends.

\section{ACKNOWLEDGEMENTS}

We thank M. Bonhivers, L. Kohl, D. Robinson, K. Matthews and K. Gull for providing various plasmids and antibodies. We are thankful to the IP Biolmaging Plateforme for providing access to their equipment. We acknowledge the generous supply of tsetse pupae from the International Atomic Energy Agency, Vienna. We thank Christelle Travaillé and Aline Crouzols for their help in fly infections. We also thank M. Boshart for his critical reading of the manuscript. This work was supported by the Institut Pasteur, the Institut National pour la Santé et le Recherche Médicale (INSERM), the French Government Investissement d'Avenir ProgrammeLaboratoire d'Excellence 'Integrative Biology of Emerging Infectious Diseases' (ANR-10-LABX-62-IBEID) and the French National Agency for Scientific Research (projects ANR-14-CE14-0019-01 EnTrypa and ANR-18-CE15-0012 TrypaDerm). UTechS PBI is part of the France-Biolmaging infrastructure network (FBI) supported by the French National Research Agency (ANR-10-INSB-04; Investments for the Future), and acknowledges support from ANR/FBI and the Région Ile-de-France (program 'Domaine d'Intérêt MajeurMalinf') for the use of the Zeiss LSM 780 Elyra PS1 microscope. None of these funding sources had a direct scientific or editorial role in the present study.

\section{CONFLICT OF INTEREST}

All authors declare no financial relationships with any organisations that might have an interest in the submitted work in the previous 3 years, no other relationships or activities that could appear to have influenced the submitted work, and no other relationships or activities that could appear to have influenced the submitted work.

\section{AUTHOR CONTRIBUTIONS}

Estefanía Calvo Alvarez, Serge Bonnefoy, Philippe Bastin and Brice Rotureau designed the study. Estefanía Calvo Alvarez, Serge Bonnefoy, Fiona E. Benson and Audrey Salles performed the experiments. Estefanía Calvo Alvarez, Serge Bonnefoy, Paul G. McKean, Philippe Bastin and Brice Rotureau analysed the data. Estefanía Calvo Alvarez and Brice Rotureau wrote the manuscript. Serge Bonnefoy, Paul G. McKean and Philippe Bastin reviewed the manuscript.

\section{DATA AVAILABILITY STATEMENT}

Data available on request from the authors.

\section{ORCID}

Estefanía Calvo-Álvarez (D) https://orcid.org/0000-0001-7509-7204 Brice Rotureau (D) https://orcid.org/0000-0003-0671-8999

\section{REFERENCES}

Abeywickrema, M., Vachova, H., Farr, H., Mohr, T., Wheeler, R. J., Lai, D. H., ... Varga, V. (2019). Non-equivalence in old- and newflagellum daughter cells of a proliferative division in Trypanosoma brucei. Molecular Microbiology, 112(3), 1024-1040. https://doi.org/10. 1111/mmi.14345

Acosta-Serrano, A., Vassella, E., Liniger, M., Kunz Renggli, C., Brun, R., Roditi, I., \& Englund, P. T. (2001). The surface coat of procyclic Trypanosoma brucei: Programmed expression and proteolytic cleavage of procyclin in the tsetse fly. Proceedings of the National Academy of Sciences of the United States of America, 98(4), 1513-1518. https://doi. org/10.1073/pnas.041611698

Barquilla, A., Saldivia, M., Diaz, R., Bart, J. M., Vidal, I., Calvo, E., ... Navarro, M. (2012). Third target of rapamycin complex negatively regulates development of quiescence in Trypanosoma brucei. Proceedings of the National Academy of Sciences of the United States of America, 109(36), 14399-14404. https://doi.org/10.1073/pnas.1210465109

Bastin, P., MacRae, T. H., Francis, S. B., Matthews, K. R., \& Gull, K. (1999). Flagellar morphogenesis: Protein targeting and assembly in the paraflagellar rod of trypanosomes. Molecular and Cellular Biology, 19(12), 8191-8200.

Bertiaux, E., Morga, B., Blisnick, T., Rotureau, B., \& Bastin, P. (2018). A grow-and-lock model for the control of flagellum length in trypanosomes. Current Biology, 28(23), 3802-3814 e3803. https://doi.org/10. 1016/j.cub.2018.10.031

Bloch, M. A., \& Johnson, K. A. (1995). Identification of a molecular chaperone in the eukaryotic flagellum and its localization to the site of microtubule assembly. Journal of Cell Science, 108(Pt 11), 3541-3545.

Bringaud, F., Robinson, D. R., Barradeau, S., Biteau, N., Baltz, D., \& Baltz, T. (2000). Characterization and disruption of a new Trypanosoma brucei repetitive flagellum protein, using double-stranded RNA inhibition. Molecular and Biochemical Parasitology, 111(2), 283-297.

Broadhead, R., Dawe, H. R., Farr, H., Griffiths, S., Hart, S. R., Portman, N., ... Gull, K. (2006). Flagellar motility is required for the viability of the bloodstream trypanosome. Nature, 440(7081), 224-227. https://doi. org/10.1038/nature04541

Brun, R., \& Schonenberger, M. (1979). Cultivation and in vitro cloning or procyclic culture forms of Trypanosoma brucei in a semi-defined medium. Short communication. Acta Tropica, 36(3), 289-292.

Buisson, J., Chenouard, N., Lagache, T., Blisnick, T., Olivo-Marin, J. C., \& Bastin, P. (2013). Intraflagellar transport proteins cycle between the 
flagellum and its base. Journal of Cell Science, 126(Pt 1), 327-338. https://doi.org/10.1242/jcs.117069

Burkard, G., Fragoso, C. M., \& Roditi, I. (2007). Highly efficient stable transformation of bloodstream forms of Trypanosoma brucei. Mol Biochem Parasitol, 153(2), 220-223. https://doi.org/10.1016/j.molbiopara. 2007.02.008.

Chan, K. Y., \& Ersfeld, K. (2010). The role of the Kinesin-13 family protein TbKif13-2 in flagellar length control of Trypanosoma brucei. Molecular and Biochemical Parasitology, 174(2), 137-140. https://doi.org/10. 1016/j.molbiopara.2010.08.001

Czichos, J., Nonnengaesser, C., \& Overath, P. (1986). Trypanosoma brucei: Cis-aconitate and temperature reduction as triggers of synchronous transformation of bloodstream to procyclic trypomastigotes in vitro. Experimental Parasitology, 62(2), 283-291. https://doi.org/10.1016/ 0014-4894(86)90033-0

Dacheux, D., Landrein, N., Thonnus, M., Gilbert, G., Sahin, A., Wodrich, H., ... Bonhivers, M. (2012). A MAP6-related protein is present in protozoa and is involved in flagellum motility. PLoS ONE, 7(2), e31344. https:// doi.org/10.1371/journal.pone.0031344

Dean, S., Marchetti, R., Kirk, K., \& Matthews, K. R. (2009). A surface transporter family conveys the trypanosome differentiation signal. Nature, 459(7244), 213-217. https://doi.org/10.1038/nature07997

Engstler, M., \& Boshart, M. (2004). Cold shock and regulation of surface protein trafficking convey sensitization to inducers of stage differentiation in Trypanosoma brucei. Genes \& Development, 18(22), 27982811. https://doi.org/10.1101/gad.323404

Field, M. C., \& Carrington, M. (2009). The trypanosome flagellar pocket. Nature Reviews. Microbiology, 7(11), 775-786. https://doi.org/10. 1038/nrmicro2221

Fort, C., Bonnefoy, S., Kohl, L., \& Bastin, P. (2016). Intraflagellar transport is required for the maintenance of the trypanosome flagellum composition but not its length. Journal of Cell Science, 129(15), 3026-3041. https://doi.org/10.1242/jcs.188227

Gibson, W., \& Bailey, M. (2003). The development of Trypanosoma brucei within the tsetse fly midgut observed using green fluorescent trypanosomes. Kinetoplastid Biology and Disease, 2(1), 1.

Heddergott, N., Kruger, T., Babu, S. B., Wei, A., Stellamanns, E., Uppaluri, S., ... Engstler, M. (2012). Trypanosome motion represents an adaptation to the crowded environment of the vertebrate bloodstream. PLoS Pathogens, 8(11), e1003023. https://doi.org/10.1371/ journal.ppat.1003023

Hirumi, H., \& Hirumi, K. (1989). Continuous cultivation of Trypanosoma brucei blood stream forms in a medium containing a low concentration of serum protein without feeder cell layers. The Journal of Parasitology, 75(6), 985-989.

Hoog, J. L., Lacomble, S., O'Toole, E. T., Hoenger, A., McIntosh, J. R., \& Gull, K. (2014). Modes of flagellar assembly in Chlamydomonas reinhardtii and Trypanosoma brucei. elife, 3, e01479. https://doi.org/10. 7554/eLife.01479

Hughes, L., Towers, K., Starborg, T., Gull, K., \& Vaughan, S. (2013). A cellbody groove housing the new flagellum tip suggests an adaptation of cellular morphogenesis for parasitism in the bloodstream form of Trypanosoma brucei. Journal of Cell Science, 126(24), 5748-5757. https://doi.org/10.1242/jcs.139139

Kelly, S., Reed, J., Kramer, S., Ellis, L., Webb, H., Sunter, J., ... Carrington, M. (2007). Functional genomics in Trypanosoma brucei: A collection of vectors for the expression of tagged proteins from endogenous and ectopic gene loci. Molecular and Biochemical Parasitology, 154(1), 103-109. https://doi.org/10.1016/j.molbiopara.2007. 03.012

Kohl, L., Robinson, D., \& Bastin, P. (2003). Novel roles for the flagellum in cell morphogenesis and cytokinesis of trypanosomes. The EMBO Journal, 22(20), 5336-5346. https://doi.org/10.1093/emboj/cdg518

Kohl, L., Sherwin, T., \& Gull, K. (1999). Assembly of the paraflagellar rod and the flagellum attachment zone complex during the Trypanosoma brucei cell cycle. The Journal of Eukaryotic Microbiology, 46(2), 105-109.

Langousis, G., \& Hill, K. L. (2014). Motility and more: The flagellum of Trypanosoma brucei. Nature Reviews. Microbiology, 12(7), 505-518. https://doi.org/10.1038/nrmicro3274

Le Ray, D., Barry, J. D., Easton, C., \& Vickerman, K. (1977). First tsetse fly transmission of the "AnTat" serodeme of Trypanosoma brucei. Annales de la Société Belge de Médecine Tropicale, 57(4-5), 369-381.

Lemos, M., Mallet, A., Bertiaux, E., Imbert, A., Rotureau, B., \& Bastin, P. (2020). Timing and original features of flagellum assembly in trypanosomes during development in the tsetse fly. Parasites \& Vectors, 13(1), 169. https://doi.org/10.1186/s13071-020-04026-0

Liu, W., Apagyi, K., McLeavy, L., \& Ersfeld, K. (2010). Expression and cellular localisation of calpain-like proteins in Trypanosoma brucei. Molecular and Biochemical Parasitology, 169(1), 20-26. https://doi.org/10.1016/ j.molbiopara.2009.09.004

MacGregor, P., Szoor, B., Savill, N. J., \& Matthews, K. R. (2012). Trypanosomal immune evasion, chronicity and transmission: An elegant balancing act. Nature Reviews. Microbiology, 10(6), 431-438. https://doi.org/10.1038/nrmicro2779

MacLeod, E. T., Maudlin, I., Darby, A. C., \& Welburn, S. C. (2007). Antioxidants promote establishment of trypanosome infections in tsetse. Parasitology, 134(Pt 6), 827-831. https://doi.org/10.1017/ S0031182007002247

Oberholzer, M., Langousis, G., Nguyen, H. T., Saada, E. A., Shimogawa, M. M., Jonsson, Z. O., ... Hill, K. L. (2011). Independent analysis of the flagellum surface and matrix proteomes provides insight into flagellum signaling in mammalian-infectious Trypanosoma brucei. Molecular \& Cellular Proteomics, 10(10), M111 010538. https:// doi.org/10.1074/mcp.M111.010538

Ooi, C. P., \& Bastin, P. (2013). More than meets the eye: Understanding Trypanosoma brucei morphology in the tsetse. Frontiers in Cellular and Infection Microbiology, 3, 71. https://doi.org/10.3389/fcimb.2013. 00071

Ooi, C. P., Rotureau, B., Gribaldo, S., Georgikou, C., Julkowska, D., Blisnick, T., ... Bastin, P. (2015). The Flagellar arginine kinase in Trypanosoma brucei is important for infection in tsetse flies. PLoS ONE, 10(7), e0133676. https://doi.org/10.1371/journal.pone.0133676

Peacock, L., Kay, C., Bailey, M., \& Gibson, W. (2018). Shape-shifting trypanosomes: Flagellar shortening followed by asymmetric division in Trypanosoma congolense from the tsetse proventriculus. PLoS Pathogens, 14(5), e1007043. https://doi.org/10.1371/journal.ppat.1007043

Pedersen, L. B., Geimer, S., \& Rosenbaum, J. L. (2006). Dissecting the molecular mechanisms of intraflagellar transport in Chlamydomonas. Current Biology, 16(5), 450-459. https://doi.org/10.1016/j.cub.2006. 02.020

Pereira, C. A., Alonso, G. D., Torres, H. N., \& Flawia, M. M. (2002). Arginine kinase: A common feature for management of energy reserves in African and American flagellated trypanosomatids. The Journal of Eukaryotic Microbiology, 49(1), 82-85. https://doi.org/10.1111/j.1550-7408. 2002.tb00346.x

Reiter, J. F., Blacque, O. E., \& Leroux, M. R. (2012). The base of the cilium: Roles for transition fibres and the transition zone in ciliary formation, maintenance and compartmentalization. EMBO Reports, 13(7), 608618. https://doi.org/10.1038/embor.2012.73

Robinson, D. R., \& Gull, K. (1991). Basal body movements as a mechanism for mitochondrial genome segregation in the trypanosome cell cycle. Nature, 352(6337), 731-733. https://doi.org/10.1038/352731a0

Roditi, I., Carrington, M., \& Turner, M. (1987). Expression of a polypeptide containing a dipeptide repeat is confined to the insect stage of Trypanosoma brucei. Nature, 325(6101), 272-274. https://doi.org/10. 1038/325272a0

Roditi, I., Schumann, G., \& Naguleswaran, A. (2016). Environmental sensing by African trypanosomes. Current Opinion in Microbiology, 32, 26-30. https://doi.org/10.1016/j.mib.2016.04.011 
Rotureau, B., Blisnick, T., Subota, I., Julkowska, D., Cayet, N., Perrot, S., \& Bastin, P. (2014). Flagellar adhesion in Trypanosoma brucei relies on interactions between different skeletal structures in the flagellum and cell body. Journal of Cell Science, 127(Pt 1), 204-215. https://doi.org/ 10.1242/jcs.136424

Rotureau, B., Morales, M. A., Bastin, P., \& Spath, G. F. (2009). The flagellum-MAP kinase connection in Trypanosomatids: A key sensory role in parasite signaling and development?Cellular Microbiology, 11(5), 710-718. https://doi.org/10.1111/j.1462-5822.2009. 01295.x

Rotureau, B., Ooi, C. P., Huet, D., Perrot, S., \& Bastin, P. (2014). Forward motility is essential for trypanosome infection in the tsetse fly. Cellular Microbiology, 16(3), 425-433. https://doi.org/10.1111/cmi.12230

Rotureau, B., Subota, I., \& Bastin, P. (2011). Molecular bases of cytoskeleton plasticity during the Trypanosoma brucei parasite cycle. Cellular Microbiology, 13(5), 705-716. https://doi.org/10.1111/j.1462-5822. 2010.01566.x

Rotureau, B., Subota, I., Buisson, J., \& Bastin, P. (2012). A new asymmetric division contributes to the continuous production of infective trypanosomes in the tsetse fly. Development, 139(10), 1842-1850. https:// doi.org/10.1242/dev.072611

Rotureau, B., \& Van Den Abbeele, J. (2013). Through the dark continent: African trypanosome development in the tsetse fly. Frontiers in Cellular and Infection Microbiology, 3, 53. https://doi.org/10.3389/fcimb.2013. 00053

Saada, E. A., Kabututu, Z. P., Lopez, M., Shimogawa, M. M., Langousis, G., Oberholzer, M., ... Hill, K. L. (2014). Insect stage-specific receptor adenylate cyclases are localized to distinct subdomains of the Trypanosoma brucei flagellar membrane. Eukaryotic Cell, 13, 10641076. https://doi.org/10.1128/EC.00019-14

Sharma, R., Peacock, L., Gluenz, E., Gull, K., Gibson, W., \& Carrington, M. (2008). Asymmetric cell division as a route to reduction in cell length and change in cell morphology in trypanosomes. Protist, 159(1), 137151. https://doi.org/10.1016/j.protis.2007.07.004

Sherwin, T., \& Gull, K. (1989). The cell division cycle of Trypanosoma brucei brucei: Timing of event markers and cytoskeletal modulations. Philosophical Transactions of the Royal Society of London. Series B, Biological Sciences, 323(1218), 573-588.

Sherwin, T., Schneider, A., Sasse, R., Seebeck, T., \& Gull, K. (1987). Distinct localization and cell cycle dependence of $\mathrm{COOH}$ terminally tyrosinolated alpha-tubulin in the microtubules of Trypanosoma brucei brucei. The Journal of Cell Biology, 104(3), 439-446.

Shimogawa, M. M., Ray, S. S., Kisalu, N., Zhang, Y., Geng, Q., Ozcan, A., \& Hill, K. L. (2018). Parasite motility is critical for virulence of African trypanosomes. Scientific Reports, 8(1), 9122. https://doi.org/10.1038/ s41598-018-27228-0

Smith, T. K., Bringaud, F., Nolan, D. P., \& Figueiredo, L. M. (2017). Metabolic reprogramming during the Trypanosoma brucei life cycle. F1000Research, 6. https://doi.org/10.12688/f1000research. 10342.2

Subota, I., Julkowska, D., Vincensini, L., Reeg, N., Buisson, J., Blisnick, T., ... Bastin, P. (2014). Proteomic analysis of intact flagella of procyclic Trypanosoma brucei cells identifies novel flagellar proteins with unique sub-localisation and dynamics. Molecular \& Cellular Proteomics, 13, 1769-1786. https://doi.org/10.1074/mcp.M113.033357

Sunter, J. D., Benz, C., Andre, J., Whipple, S., McKean, P. G., Gull, K., ... Lukes, J. (2015). Modulation of flagellum attachment zone protein
FLAM3 and regulation of the cell shape in Trypanosoma brucei life cycle transitions. Journal of Cell Science, 128(16), 3117-3130. https:// doi.org/10.1242/jcs.171645

Szoor, B., Ruberto, I., Burchmore, R., \& Matthews, K. R. (2010). A novel phosphatase cascade regulates differentiation in Trypanosoma brucei via a glycosomal signaling pathway. Genes \& Development, 24(12), 1306-1316. https://doi.org/10.1101/gad.570310

Taylor, A. E., \& Godfrey, D. G. (1969). A new organelle of bloodstream salivarian trypanosomes. The Journal of Protozoology, 16(3), 466-470. https://doi.org/10.1111/j.1550-7408.1969.tb02302.x

Tetley, L., \& Vickerman, K. (1985). Differentiation in Trypanosoma brucei: Host-parasite cell junctions and their persistence during acquisition of the variable antigen coat. Journal of Cell Science, 74, 1-19.

Urbaniak, M. D., Martin, D. M., \& Ferguson, M. A. (2013). Global quantitative SILAC phosphoproteomics reveals differential phosphorylation is widespread between the procyclic and bloodstream form lifecycle stages of Trypanosoma brucei. Journal of Proteome Research, 12(5), 2233-2244. https://doi.org/10.1021/pr400086y

Urwyler, S., Studer, E., Renggli, C. K., \& Roditi, I. (2007). A family of stagespecific alanine-rich proteins on the surface of epimastigote forms of Trypanosoma brucei. Molecular Microbiology, 63(1), 218-228. https:// doi.org/10.1111/j.1365-2958.2006.05492.x

Van Den Abbeele, J., Claes, Y., van Bockstaele, D., Le Ray, D., \& Coosemans, M. (1999). Trypanosoma brucei spp. development in the tsetse fly: Characterization of the post-mesocyclic stages in the foregut and proboscis. Parasitology, 118(5), 469-478.

Varga, V., Moreira-Leite, F., Portman, N., \& Gull, K. (2017). Protein diversity in discrete structures at the distal tip of the trypanosome flagellum. Proceedings of the National Academy of Sciences of the United States of America, 114(32), E6546-E6555. https://doi.org/10.1073/ pnas.1703553114

Woodward, R., Carden, M. J., \& Gull, K. (1995). Immunological characterization of cytoskeletal proteins associated with the basal body, axoneme and flagellum attachment zone of Trypanosoma brucei. Parasitology, 111(Pt 1), 77-85.

Woodward, R., \& Gull, K. (1990). Timing of nuclear and kinetoplast DNA replication and early morphological events in the cell cycle of Trypanosoma brucei. Journal of Cell Science, 95(1), 49-57.

Woolley, D., Gadelha, C., \& Gull, K. (2006). Evidence for a slidingresistance at the tip of the trypanosome flagellum. Cell Motility and the Cytoskeleton, 63(12), 741-746. https://doi.org/10.1002/cm.20159

\section{SUPPORTING INFORMATION}

Additional supporting information may be found online in the Supporting Information section at the end of this article.

How to cite this article: Calvo-Álvarez, E., Bonnefoy, S., Salles, A., Benson, F. E., McKean, P. G., Bastin, P., \& Rotureau, B. (2021). Redistribution of FLAgellar Member 8 during the trypanosome life cycle: Consequences for cell fate prediction. Cellular Microbiology, 23(9), e13347. https://doi.org/10. 1111/cmi.13347 